\title{
Metamorphic evolution and P-T path of gneisses from Goiás Magmatic Arc in southwestern Goiás State
}

\author{
Evolução metamórfica e trajetória P-T de gnaisses do Arco \\ Magmático de Goiás no sudoeste do Estado de Goiás
}

Guillermo R. B. Navarroํ, Antenor Zanardo ${ }^{1}$, Fabiano Tomazini da Conceição ${ }^{2 *}$

\begin{abstract}
In the southwestern Goiás State, the rocks of the Goiás Magmatic Arc present mineral assemblages typical of the amphibolite facies. Thermobarometric calculations show that $\mathrm{P}$ and $\mathrm{T}$ conditions for associations involving amphibole + biotite + kyanite + staurolite + garnet + plagioclase + muscovite, biotite + garnet + plagioclase + muscovite, amphibole + biotite + garnet + plagioclase, belong to the middle amphibolite facies in the staurolite-kyanite field, reaching temperatures and pressure higher than $600^{\circ} \mathrm{C}$ and $7 \mathrm{kbar}$, respectively. The P-T path is clockwise and the values obtained by thermobarometric calculations are interpreted as a retrometamorphic reequilibrium in the amphibolite facies.
\end{abstract}

KEYWORDS: Brasília Belt; Goiás Magmatic Arc; mineral chemistry; thermobarometry.

\section{INTRODUCTION}

The Brasília Belt is an orogenic belt that records a complete Wilson Cycle, including pataformal metasedimentary sequences of passive margin and deep marine sequences (as in Barbosa et al. 1970, Marini et al. 1984a e 1984b, Fuck et al. 1993, Dardenne 2000, Valeriano et al. 2004), metasedimentary sequences of fore arc type (Navarro et al. 2012); alpine-type metamafic/metaultramafic rocks, recognized as an ophiolitic mélange (as in Drake Jr. 1980, Nilson 1984, Brod et al. 1991, Strieder \& Nilson 1992), and magmatic arcs (Pimentel \& Fuck 1991 e 1992, Fuck et al. 1993, Pimentel et al. 2000a e 2004, Dardenne 2000), probably descendant of the consumption of the oceanic and São Franciscana plates.
RESUMO: No sudoeste do estado de Goiás, as rochas do Arco Magmático de Goiás apresentam associaçōes minerais típicas da fácies anfibolito. Cálculos termobarométricos mostram que as condiçôes $P$ e T para associaçóes envolvendo anfibólio + biotita + cianita + estaurolita + granada + plagioclásio + muscovita, biotita + granada + plagioclásio + muscovita, anfibólio + biotita + granada + plagioclásio são da fácies anfibolito médio, na zona da cianita-estaurolita, atingindo temperaturas e pressöes maiores que $600^{\circ} \mathrm{C} \mathrm{e} 7 \mathrm{kbar}$, respectivamente. A trajetória P e Té horária, e os valores obtidos nos cálculos termobarométricos são interpretados como um reequilíbrio retrometamórfico de fácies anfibolito.

PALAVRAS-CHAVE: Faixa Brasilia; Arco Magmático de Goiás; química mineral; termobarometria.

It is possible to summarize the metamorphism in the Brasília Belt as a variation from greenschist facies to amphibolite facies, reaching granulite or eclogite facies in Goiás and Minas Gerais (Zanardo et al. 1990 e 1996, Zanardo 1992, Del Lama et al. 1994, Winge 1995, Campos Neto \& Caby 1999 e 2000, Moraes et al. 2002, Navarro et al. 2009, 2011). The oscillation of the metamorphic assemblages indicates the inversion of the metamorphism in some regions (Oliveira et al. 1983, Simóes et al. 1988, Simóes 1995, Luvizotto 2003), and that it reached its peak during Neoproterozoic (Fischel et al. 1998, Piuzana et al. 2003). There are few detailed works about the metamorphism in the southwestern region of the Brasília Belt. Thus, the present work intended to contribute for the characterization of the metamorphic

\footnotetext{
${ }^{1}$ Departamento de Petrologia e Metalogenia - DPM, Universidade Estadual Paulista “Júlio de Mesquita Filho” - UNESP, Rio Claro (SP), Brasil. E-mail: navarro@rc.unesp.br azanardo@rc.unesp.br

${ }^{2}$ Departamento de Planejamento Territorial e Geoprocessamento - DEPLAM, Universidade Estadual Paulista “Júlio de Mesquita Filho” - UNESP, Rio Claro (SP), Brasil. E-mail: ftomazini@rc.unesp.br

Manuscrito ID 26053. Recebido em: 05/03/2012. Aprovado em: 21/01/2013
} 
conditions of Goiás Magmatic Arc in the Pontalina region, southwest of Goiás State.

\section{LOCAL GEOLOGY}

The investigated area is situated in the meridional portion of the Brasilia Belt, nearby Pontalina city, in the limit between metasediments of the Internal Domain in the Brasilia Belt, on the east, and the sequence of supracrustal rocks (Anicuns-Itaberaí Metavolcanossedimentary Sequence), on the west (Fig. 1). In this area, the Goiás Magmatic Arc is grouped by the informal name of Gneissic-Metasedimentary Terranes, which Navarro \& Zanardo (2007a) subdivided into (a) Gneissic Unit and (b) Metasedimentary Unit (Fig. 2).

The Gneissic Unit consists of a belt of several types of gneisses that includes muscovite gneisses, biotite-muscovite gneisses, hornblende gneisses, hornblende-biotite gneisses with or without porphyritic texture, usually quartzose and epidote-rich. They exhibit varying mylonitization degrees, predominating terms containing biotite and muscovite. Chemically, these gneisses are calcic to calc-alkaline in composition, metaluminous to peraluminous, low in alkalis, with $\mathrm{Ba}$-, $\mathrm{Sr}$-, $\mathrm{K}$ - and $\mathrm{Rb}$-enrichment in relation to $\mathrm{Nb}, \mathrm{Y}, \mathrm{Zr}$ and ETR, and present negative anomalies of $\mathrm{Ti}$ e $\mathrm{Nb}$, similar to the composition of magmas generated in magmatic arcs (Navarro \& Zanardo 2007a). Isotopic data Sm-Nd of these gneisses display model ages $\mathrm{T}_{\mathrm{DM}}$ between 0.90 and $1.46 \mathrm{Ga}$ and isotopic compositions ${ }^{147} \mathrm{Sm} /{ }^{144} \mathrm{Nd}$ between $0.0881-0.1383$ and ${ }^{143} \mathrm{Nd} /{ }^{144} \mathrm{Nd}$ between 0.512173 - 0.512600 (Navarro \& Zanardo 2007a).

The Metasedimentary Unit contains pelitic to psamo-pelitic metasedimentary rocks (muscovite schist, muscovite-quartz schist, quartzite, ferruginous quartzite etc.) intercalated by quartz gneisses (muscovite gneisses, biotite-muscovite gneisses, usually with strong muscovitization and epidoto-rich). These are orto- or paraderivated, mainly peraluminous in composition, with calc-alkaline affinity and normative granitic mineralogy,

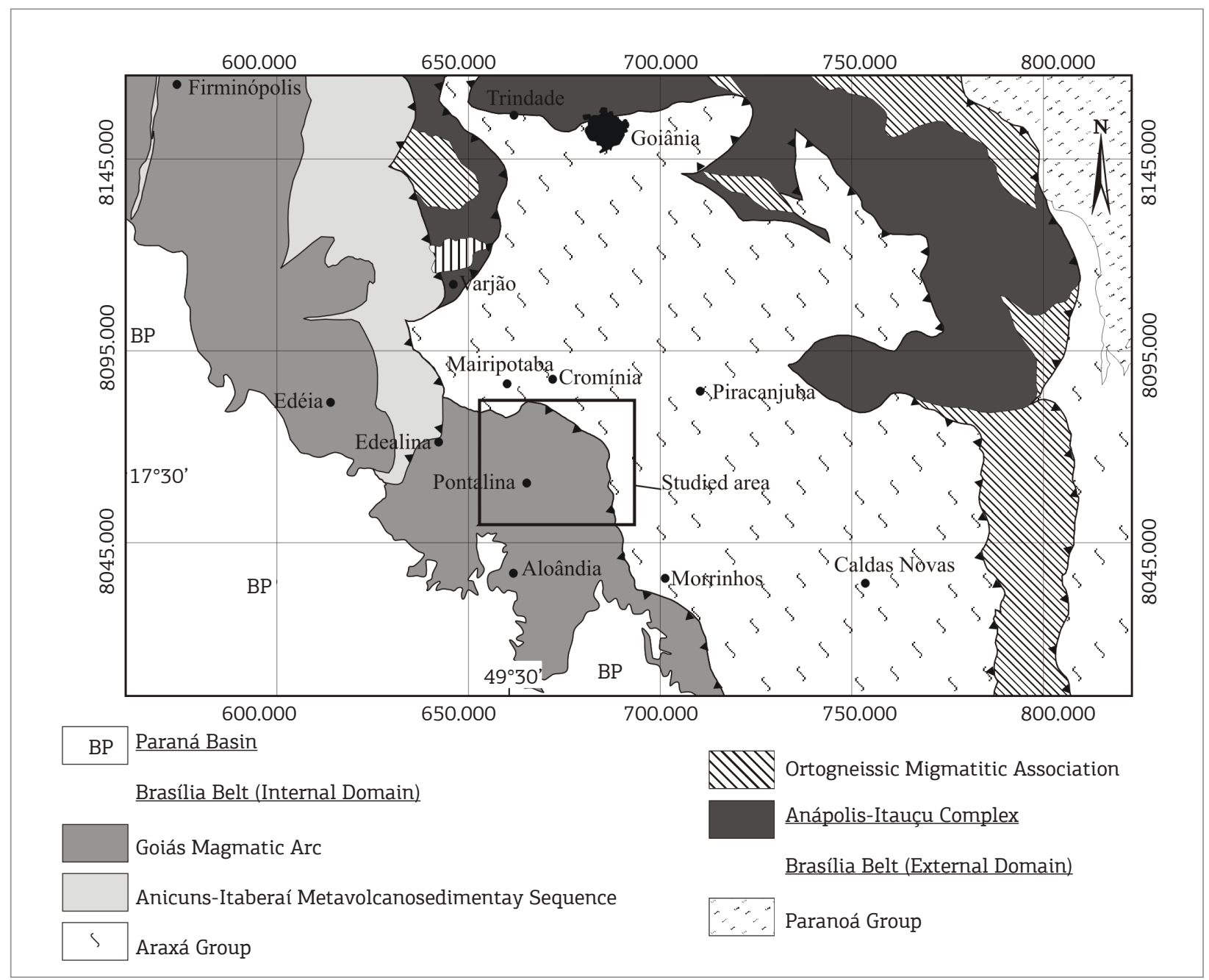

Figure 1. Schematic geological map, showing the location of the studied area. 
exhibiting wide variation in the content of major and trace elements, with relatively low to moderate $\mathrm{Y}$ and $\mathrm{Nb}$ content, and high $\mathrm{Ba}, \mathrm{Sr}$ and $\mathrm{Rb}$ content, which are related to an island arc environment, probably being inserted in the same geological context of the Gneissic Unit (Navarro \& Zanardo 2007b).

Metamafic rocks (amphibolites, garnet amphibolite, garnet-amphibole schist and amphibole schist) occur as lenses of decimetric to decametric thickness and metric to hectometric extents, oriented in the direction of the banding of the host rocks, in both units, being less frequent in the Metasedimentary Unit. These are ortoderivated rocks and have chemical composition of basic rocks, predominating compositions of sub-alkaline basalts and andesitic basalts; they also present tholeiític affinity, subalkaline to alkaline, with geochemical characteristics similar to oceanic basalts, especially the E-MORB type and arc basalts (Navarro \& Zanardo 2006; Navarro et al. 2007). Isotopic data $\mathrm{Sm}-\mathrm{Nd}$ on amphibolites of this region show model ages $T_{D M}$ between 1.0 and 1.23 and isotopic composition ${ }^{147} \mathrm{Sm} /{ }^{144} \mathrm{Nd}$ ranging between $0.106-0.122$ and ${ }^{143} \mathrm{Nd} /{ }^{144} \mathrm{Nd}$ between $0.512164-0.512445$ (Navarro \& Zanardo 2006; Navarro et al. 2007). Both sequences contain metaultramafic rock bodies (chlorite schist, talc schist and less often serpentinites), and the most important is the constituent of the Morro Dois Irmáos (Gneissic Unit).
A well-developed foliation is the main structure, usually parallel to the compositional banding. This Sn foliation is characterized by oriented crystals of phyllossilicates, elongated quartz, epidote, hornblende, opaque minerals constituting trails and polycrystalline aggregates in lenticular or fusiform shapes. This fabric defines schistosity, in various development degrees, reaching sometimes blastomylonitic features. Locally, there is a well-developed schistosity, named as Sn-1 foliation, parallel to a milimetric to metric compositional banding, constituted of variations between lepidoblastic and granoblastic portions, and by a centimetric to metric intercalation of several litotypes. This schistosity $\mathrm{Sn}-1$ was folded and transposed by the main foliation (Sn). The Sn foliation is oriented in N-S to NNW/ SSE direction and has a NW dip with low to moderate angle $(S n=290 / 26)$. In the northern region, this foliation is oriented roughly in the E-W direction, with a low to moderate dip angle to SW $(\mathrm{Sn}=214 / 17)$. In association with Sn, a mineral and/or stretching lineation is recognized, in E-W direction (256/09) and low dip to W. Cinematic indicators suggest direction of transport from $\mathrm{W}$ to $\mathrm{E}$.

\section{Metamorphism}

The rocks described in the investigated area were affected by a single metamorphic event, in which later parageneses and mineral assemblages typically belong to the amphibolite facies, and the earlier ones belong to greenschist

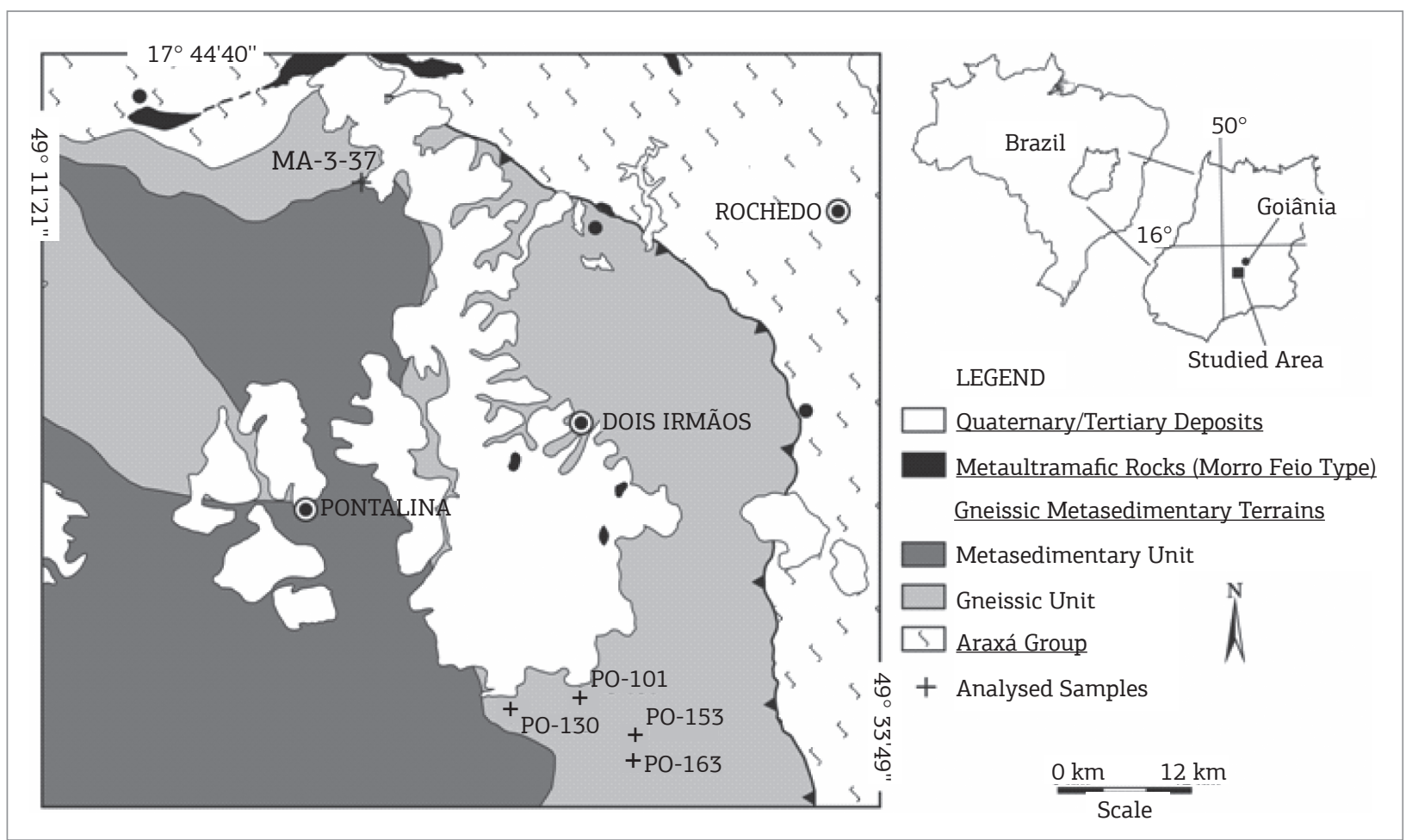

Figure 2. Schematic geological map of the studied area, showing the location of the sampling points. 
facies, being generated in the last stages of retrometamorphism. Metamorphic peak (M1) occurred in amphibolite facies conditions and is emphasized by amphibole + plagioclase and garnet + amphibole + plagioclase associations, with rutile and ilmenite as the main associated oxides (in metamafic rocks). Except for a single sample of metamafic rock with a plagioclase + clinopyroxene + garnet + quartz + ortopyroxene + rutile + ilmenite association that represents granulite facies paragenesis, there are no petrographic, textural or mineralogical evidences that this region reached transition between amphibolite and granulite facies. This sample has an anomalous assemblage (granulite facies) when compared to the other rocks recognized in this region, and it is not inserted in the regional metamorphic context, probably corresponding to a tectonic enclave of another geological unit (Navarro et al. 2011). Thermobarometric calculation shows $\mathrm{P}$ and $\mathrm{T}$ conditions for amphibole + plagioclase and plagioclase + garnet + amphibole, in metamafic rocks, in middle to upper amphibolite facies, in kyanite stability field, and that it reached temperatures slightly above $700^{\circ} \mathrm{C}$, not exceeding $775^{\circ} \mathrm{C}$, at moderate pressure ( $-10 \mathrm{kbar})$ (Navarro et al. 2011).

In the gneisses, amphibolite facies conditions are expressed by the presence of plagioclase (oligoclase-andesine) + potassic feldspar (ortoclase and microcline) + biotite, plagioclase + biotite + hornblende, locally associated with garnet and rutile, and the presence of muscovite. Thus, in some greatly aluminous types, muscovite also shows stability in the metamorphic peak. This assemblage indicates that the minimum temperatures were about 600 to $750^{\circ} \mathrm{C}$ (kyanite-staurolite stability field) in a pressure regime compatible with or greater than barrowian. The presence of gneissified mobilized quartz-plagioclase indicates minimum temperature above $650^{\circ} \mathrm{C}$ at the metamorphic peak.

Greenschist facies retrometamorphism is characterized by the presence of minerals reequilibrated or neoformed in amphibolite to greenschist facies, involving the transformation of garnet and amphibole to biotite and chlorite; formation of ilmenite and titanite; appearance of epidote, albite and white micas, interstitially and above the plagioclase; clear albite coronas at the rims of saussuritized plagioclase; formation of actinolite along the rim of hornblende; sericitization of kyanite and staurolite, and serpentinization of olivine. Lower-grade retrometamorphic associations are interpreted as a record of the reequilibrium during exhumation by erosion and cooling, which started in the late stages of the deformation phase that generated the main foliation (Sn) and followed late deformation (post-Dn). Retrometamorphic transformations, catalyzed by fluid circulation during later stages in the development of main foliation, are observed with higher or lower intensities on rocks, especially in the Metasedimentary Unit, in which saussuritization of plagioclases is frequent, besides formation of yellow-green epidote, as euhedral to anhedral crystals, scattered or constituting trails and veinlets, accordant or not with the banding or foliation, and muscovite forming relatively wide crystals $(2-3 \mathrm{~mm})$, often without preferred orientation, inside plagioclase crystals or interstitially to minerals as plagioclase, potassic feldspar, amphibole, among others.

\section{MINERAL CHEMISTRY}

Minerals that define paragenesis at the metamorphic peak were analyzed on Electron Microprobe Laboratory at Geoscience Institute of Universidade de São Paulo (USP), using CAMECA SX50, under conditions of $20 \mathrm{kV}$ e $25 \mathrm{nA}$. The analysis was executed with an electron bean ranging from 1 to $5 \mu$, and the ionic microprobe was calibrated with natural patterns with known concentrations. Location of the five sampling points is represented on Fig. 2. Two to five grains were analyzed for each section, from two to three different domains, with analysis of cores and rims. In the garnet grains, there were executed, in compositional line traverses, 10 to 20 analyses each. Table 1 summarizes the observed mineral associations that were utilized for calculating the P-T conditions.

\section{Amphibole}

Chemical analyses of amphibole crystals from sample MA-3-37 are shown in Tab. 2 and indicate a variation from magnesiohastingsite to ferropargasite (Fig. 3), in which chemical zonation is not present. They exhibit Ti of 0.003 to 0.054 atoms per formulae unit (a.p.f.u.), $1.816-2.024$ a.p.f.u. of $\mathrm{Al}^{\mathrm{IV}}$ and $1.074-1.253$ a.p.f.u. of $\mathrm{Al}^{\mathrm{VI}}$. $\mathrm{Na}$ and $\mathrm{K}$ content is low, varying respectively from 0.621 to 0.732 and $0.064-0.081$ a.p.f.u., and Ca ranges from 1.589 to 1.687 a.p.f.u.; $\mathrm{Na}(\mathrm{M} 4)$ content is also low $(0.184-0.304$ a.p.f.u.). In sample PO-101, amphibole crystals present two distinct compositions (Tab. 2 and Fig. 3). The rims of the crystal show composition of tschermakite, with $\mathrm{Ti}$ of 0.01 a.p.f.u., $\mathrm{Al}^{\mathrm{IV}}$ from 0.12 to 1.37 a.p.f.u. and $\mathrm{Al}^{\mathrm{VI}}$ from 0.07 to 0.86 a.p.f.u., while the cores exhibit a cummingtonite composition, with Ti from 0.01 to 0.06 a.p.f.u., $\mathrm{Al}^{\mathrm{IV}}$ from 1.37 to 1.78 a.p.f.u. and $\mathrm{Al}^{\mathrm{VI}}$ from 0.86 to 1.07 a.p. f.u. In tschermakite, $\mathrm{Na}$ content varies between 0.224 and 0.556 a.p.f.u., $\mathrm{K}$ varies from 0.01 to 0.051 a.p.f.u. and $\mathrm{Ca}$ ranges between $1.441-1.909$ a.p.f.u.; $\mathrm{Na}$ (M4) content is low $(0.0490-0.22$ a.p.f.u.). 
Guillermo R. B. Navarro et al.

Table 1. Mineralogical assemblages present in the samples selected for thermobarometry

\begin{tabular}{c|c|c|c|c|c|c|c}
\hline Sample & Amphibole & Biotite & Kyanite & Staurolite & Feldspar & Garnet & Muscovite \\
\hline MA-3-37 & $\mathrm{X}$ & $\mathrm{X}$ & $\mathrm{X}$ & $\mathrm{X}$ & $\mathrm{X}$ & $\mathrm{X}$ & $\mathrm{X}$ \\
\hline PO-153 & & $\mathrm{X}$ & & & $\mathrm{X}$ & $\mathrm{X}$ & $\mathrm{X}$ \\
\hline PO-163 & & $\mathrm{X}$ & & & $\mathrm{X}$ & $\mathrm{X}$ & $\mathrm{X}$ \\
\hline PO-101 & $\mathrm{X}$ & $\mathrm{X}$ & & & $\mathrm{X}$ & $\mathrm{X}$ & \\
\hline PO-130 & & $\mathrm{X}$ & & & $\mathrm{X}$ & $\mathrm{X}$ & $\mathrm{X}$ \\
\hline
\end{tabular}

Table 2. Representative chemical analyses of amphibole crystals

\begin{tabular}{|c|c|c|c|c|c|c|c|c|c|c|c|c|c|}
\hline Sample & $\begin{array}{l}\hat{n} \\
n \\
\vdots \\
\sum \\
\Sigma\end{array}$ & 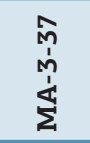 & 商 & 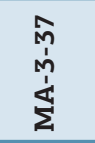 & 命 & 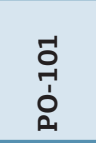 & $\begin{array}{l}-1 \\
0 \\
\text { ò } \\
\text { o }\end{array}$ & 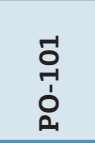 & $\begin{array}{l}-1 \\
0 \\
\text { 1 } \\
\text { o }\end{array}$ & $\begin{array}{l}-1 \\
\text { - } \\
\text { o } \\
\text { o }\end{array}$ & 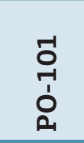 & 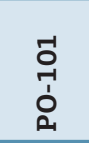 & $\begin{array}{l}\text { - } \\
\text { †े } \\
\text { ó } \\
\text { o }\end{array}$ \\
\hline position & b & $\mathrm{n}$ & $\mathrm{n}$ & b & $\mathrm{n}$ & b & $\mathbf{n}$ & b & $n$ & b & $n$ & b & $\mathbf{n}$ \\
\hline $\mathrm{SiO}_{2}$ & 41.48 & 40.74 & 40.68 & 40.21 & 40.65 & 54.25 & 44.50 & 42.99 & 54.23 & 42.21 & 53.76 & 42.57 & 53.99 \\
\hline $\mathrm{TiO}_{2}$ & 0.43 & 0.25 & 0.27 & 0.48 & 0.23 & 0.05 & 0.06 & 0.58 & 0.06 & 0.27 & 0.08 & 0.42 & 0.00 \\
\hline $\mathrm{Al}_{2} \mathrm{O}_{3}$ & 17.40 & 17.47 & 17.57 & 17.25 & 17.09 & 1.40 & 12.74 & 15.18 & 1.32 & 16.23 & 1.65 & 16.17 & 1.16 \\
\hline $\mathrm{FeO}$ & 16.88 & 16.88 & 17.44 & 17.20 & 17.02 & 20.78 & 15.16 & 16.18 & 21.56 & 17.14 & 20.80 & 16.52 & 21.24 \\
\hline $\mathrm{Cr}_{2} \mathrm{O}_{3}$ & 0.00 & 0.00 & 0.00 & 0.00 & 0.00 & 0.00 & 0.00 & 0.00 & 0.00 & 0.00 & 0.00 & 0.00 & 0.00 \\
\hline $\mathrm{MnO}$ & 0.15 & 0.14 & 0.15 & 0.17 & 0.13 & 1.98 & 0.46 & 0.64 & 1.75 & 0.73 & 1.76 & 0.63 & 2.10 \\
\hline $\mathrm{MgO}$ & 7.53 & 7.64 & 7.49 & 7.61 & 7.61 & 17.94 & 10.59 & 9.73 & 17.82 & 9.07 & 17.53 & 9.38 & 17.75 \\
\hline $\mathrm{CaO}$ & 10.37 & 10.40 & 10.35 & 9.83 & 10.10 & 0.78 & 10.35 & 9.33 & 0.77 & 8.97 & 1.51 & 9.24 & 0.59 \\
\hline $\mathrm{Na}_{2} \mathrm{O}$ & 2.15 & 2.45 & 2.44 & 2.14 & 2.25 & 0.18 & 1.60 & 1.98 & 0.13 & 2.34 & 0.19 & 2.29 & 0.15 \\
\hline $\mathrm{K}_{2} \mathrm{O}$ & 0.36 & 0.35 & 0.34 & 0.42 & 0.34 & 0.01 & 0.24 & 0.26 & 0.01 & 0.25 & 0.02 & 0.25 & 0.00 \\
\hline Sum & 96.74 & 96.33 & 96.71 & 95.30 & 95.42 & 97.36 & 95.69 & 96.86 & 97.65 & 97.20 & 97.29 & 97.47 & 96.98 \\
\hline $\mathrm{TSi}$ & 6.22 & 6.15 & 6.12 & 6.11 & 6.18 & 7.43 & 6.63 & 6.34 & 7.42 & 6.23 & 7.41 & 6.25 & 7.43 \\
\hline TAl & 1.78 & 1.85 & 1.88 & 1.89 & 1.82 & 0.14 & 1.37 & 1.66 & 0.14 & 1.77 & 0.19 & 1.75 & 0.12 \\
\hline $\mathrm{TFe}^{3+}$ & 0.00 & 0.00 & 0.00 & 0.00 & 0.00 & 0.43 & 0.00 & 0.00 & 0.44 & 0.00 & 0.41 & 0.00 & 0.45 \\
\hline TTi & 0.00 & 0.00 & 0.00 & 0.00 & 0.00 & 0.00 & 0.00 & 0.00 & 0.00 & 0.00 & 0.00 & 0.00 & 0.00 \\
\hline Sum & 8.00 & 8.00 & 8.00 & 8.00 & 8.00 & 8.00 & 8.00 & 8.00 & 8.00 & 8.00 & 8.00 & 8.00 & 8.00 \\
\hline CAl & 1.29 & 1.25 & 1.23 & 1.20 & 1.24 & 0.09 & 0.86 & 0.97 & 0.07 & 1.05 & 0.08 & 1.05 & 0.07 \\
\hline $\mathrm{CCr}$ & 0.00 & 0.00 & 0.00 & 0.00 & 0.00 & 0.00 & 0.00 & 0.00 & 0.00 & 0.00 & 0.00 & 0.00 & 0.00 \\
\hline $\mathrm{CFe}^{3+}$ & 0.30 & 0.31 & 0.35 & 0.47 & 0.38 & 0.68 & 0.46 & 0.68 & 0.71 & 0.76 & 0.71 & 0.68 & 0.69 \\
\hline CTi & 0.05 & 0.03 & 0.03 & 0.05 & 0.03 & 0.01 & 0.01 & 0.06 & 0.01 & 0.03 & 0.01 & 0.05 & 0.00 \\
\hline $\mathrm{CMg}$ & 1.68 & 1.72 & 1.68 & 1.73 & 1.72 & 3.66 & 2.35 & 2.14 & 3.63 & 2.00 & 3.60 & 2.05 & 3.64 \\
\hline $\mathrm{CFe}^{2+}$ & 1.67 & 1.68 & 1.70 & 1.54 & 1.62 & 0.45 & 1.29 & 1.11 & 0.49 & 1.13 & 0.50 & 1.13 & 0.48 \\
\hline $\mathrm{CMn}$ & 0.01 & 0.01 & 0.01 & 0.01 & 0.01 & 0.11 & 0.03 & 0.04 & 0.09 & 0.04 & 0.10 & 0.04 & 0.11 \\
\hline $\mathrm{CCa}$ & 0.00 & 0.00 & 0.00 & 0.00 & 0.00 & 0.00 & 0.00 & 0.00 & 0.00 & 0.00 & 0.00 & 0.00 & 0.00 \\
\hline Sum & 5.00 & 5.00 & 5.00 & 5.00 & 5.00 & 5.00 & 5.00 & 5.00 & 5.00 & 5.00 & 5.00 & 5.00 & 5.00 \\
\hline $\mathrm{BMg}$ & 0.00 & 0.00 & 0.00 & 0.00 & 0.00 & 0.00 & 0.00 & 0.00 & 0.00 & 0.00 & 0.00 & 0.00 & 0.00 \\
\hline $\mathrm{BFe}^{2+}$ & 0.15 & 0.14 & 0.15 & 0.18 & 0.16 & 0.82 & 0.13 & 0.21 & 0.83 & 0.23 & 0.77 & 0.22 & 0.82 \\
\hline $\mathrm{BMn}$ & 0.01 & 0.01 & 0.01 & 0.01 & 0.01 & 0.12 & 0.03 & 0.04 & 0.11 & 0.05 & 0.11 & 0.04 & 0.13 \\
\hline $\mathrm{BCa}$ & 1.67 & 1.68 & 1.67 & 1.60 & 1.64 & 0.11 & 1.65 & 1.47 & 0.11 & 1.42 & 0.22 & 1.45 & 0.09 \\
\hline $\mathrm{BNa}$ & 0.18 & 0.17 & 0.18 & 0.21 & 0.19 & 0.02 & 0.19 & 0.28 & 0.02 & 0.31 & 0.02 & 0.29 & 0.02 \\
\hline Sum & 2.00 & 2.00 & 2.00 & 2.00 & 2.00 & 1.08 & 2.00 & 2.00 & 1.07 & 2.00 & 1.13 & 2.00 & 1.06 \\
\hline $\mathrm{ACa}$ & 0.00 & 0.00 & 0.00 & 0.00 & 0.00 & 0.00 & 0.00 & 0.00 & 0.00 & 0.00 & 0.00 & 0.00 & 0.00 \\
\hline $\mathrm{ANa}$ & 0.45 & 0.55 & 0.54 & 0.42 & 0.48 & 0.03 & 0.28 & 0.29 & 0.02 & 0.36 & 0.03 & 0.37 & 0.02 \\
\hline $\mathrm{AK}$ & 0.07 & 0.07 & 0.07 & 0.08 & 0.07 & 0.00 & 0.05 & 0.05 & 0.00 & 0.05 & 0.00 & 0.05 & 0.00 \\
\hline Sum & 0.52 & 0.62 & 0.60 & 0.50 & 0.54 & 0.03 & 0.32 & 0.34 & 0.02 & 0.41 & 0.03 & 0.41 & 0.02 \\
\hline Sum & 15.52 & 15.62 & 15.60 & 15.50 & 15.54 & 14.10 & 15.32 & 15.34 & 14.09 & 15.41 & 14.16 & 15.41 & 14.08 \\
\hline
\end{tabular}

Formula proportions based on 23 oxygen atoms; $n$ : core and b: rim 


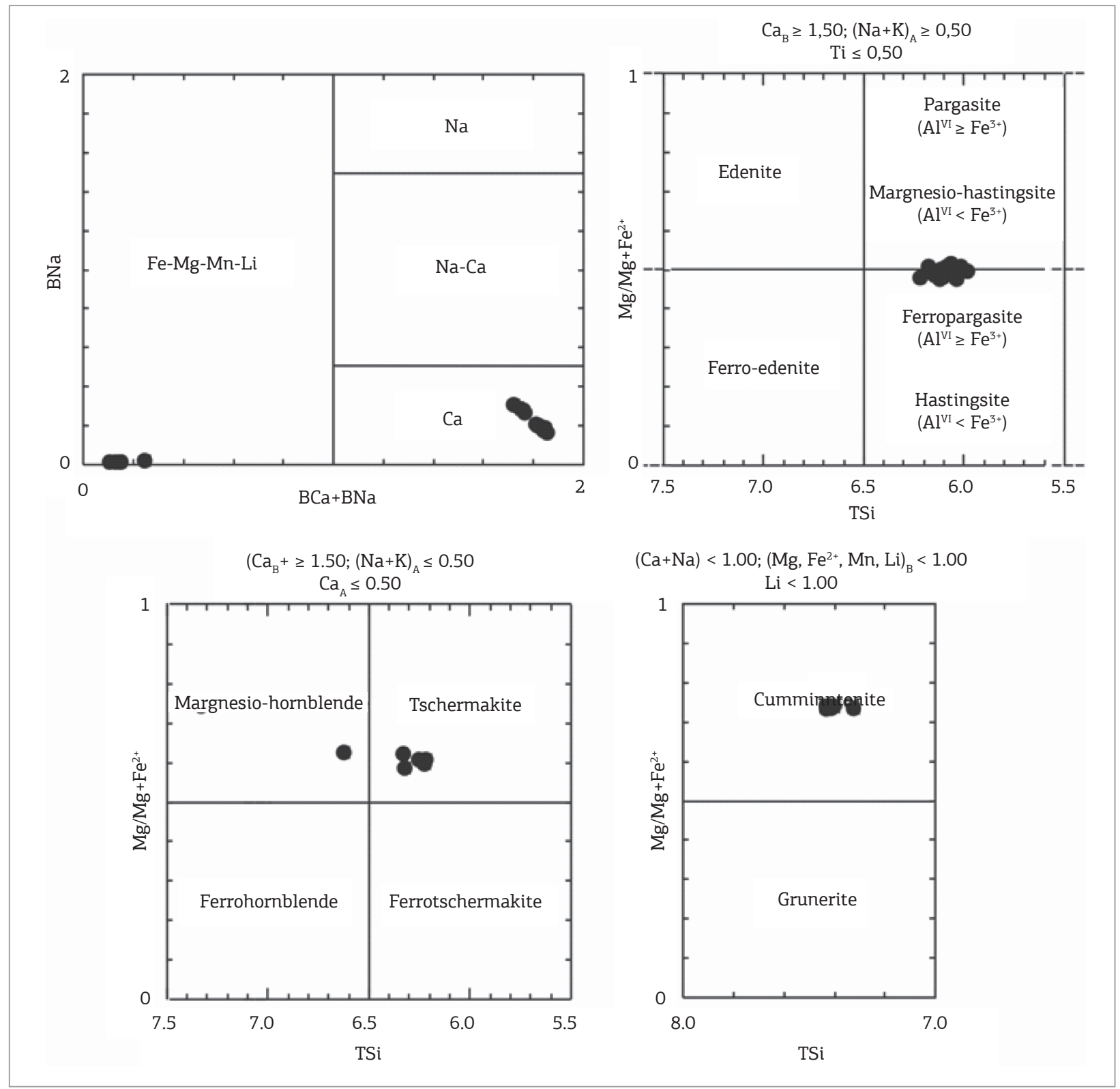

Figure 3. Classification diagram for amphiboles (Leake et al. 1997).

\section{Biotite}

Biotite found in all analyzed samples presents low Ti, with values between 0.053 and 0.129 a.p.f.u. (Tab. 3). They possess $2.596-2.792$ a.p.f.u. of $\mathrm{Al}^{\mathrm{IV}}$, and $X_{\mathrm{Mg}}$ between 0,441 and 0.652 , plotting on the eastonite field (Fig. 4). The sample PO-163 is enriched in $X_{\mathrm{Fe}}$ (ranging from 0.542 to 0.559 ) and the crystals are classified as siderophyllite.

\section{Staurolite}

Staurolite crystals were found in a single section (sample MA-3-37), as inclusions on garnet porphyroblasts and on the matrix. Staurolite crystals that occur on the matrix show $\mathrm{X}_{\mathrm{Mg}}$ content ranging between
0.01 and 0.25 , and compositional zoning is not detected. $\mathrm{Ti}$ and $\mathrm{Mg}$ contentes are low, ranging respectively between 0.090 and 0.213 and 0.003 to 0.041 a.p.f.u. (Tab. 4). Staurolite crystals included in garnet porphyroblasts present composition similar to the one observed on the matrix. Some analyses differ only in the Si content, being in most cases higher than the crystals found in the matrix (Tab. 4).

\section{Garnet}

Representative analyses of garnet grains from sample MA-3-37 are shown on Tab. 5. Samples PO-101 and PO-163 contain almandine-rich garnet crystals, followed by spessartine, pyrope and fewer grossular. On samples 
Table 3. Representative chemical analysis ofbiotite

\begin{tabular}{|c|c|c|c|c|c|c|c|c|c|c|c|c|c|c|c|}
\hline 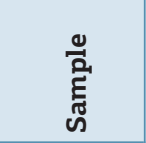 & $\begin{array}{c}\hat{n} \\
\hat{n} \\
\stackrel{\Sigma}{\Sigma}\end{array}$ & $\begin{array}{l}\hat{n} \\
\hat{n} \\
\dot{\zeta} \\
\Sigma\end{array}$ & $\frac{\hat{n}}{n}$ & $\begin{array}{l}\text { ભn } \\
\text { กิ } \\
\text { ò } \\
\text { م. }\end{array}$ & 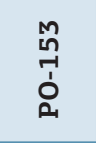 & $\begin{array}{l}\text { ભn } \\
\text { กิ } \\
\text { ò } \\
\text { م. }\end{array}$ & $\begin{array}{l}\text { No } \\
\stackrel{1}{1} \\
\text { Oे }\end{array}$ & 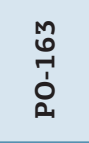 & 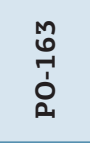 & 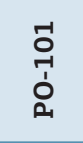 & 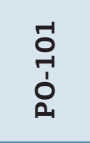 & $\begin{array}{l}\stackrel{0}{ } \\
\stackrel{1}{1} \\
\text { ò }\end{array}$ & $\begin{array}{l}\stackrel{0}{ } \\
\stackrel{1}{1} \\
\text { ò }\end{array}$ & 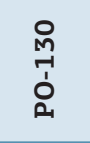 & $\begin{array}{l}\text { 을 } \\
\text { ̀ } \\
\text { ó }\end{array}$ \\
\hline position & b & $\mathrm{n}$ & b & b & b & $n$ & b & $\mathrm{n}$ & $\mathrm{n}$ & b & b & $n$ & $\mathrm{n}$ & b & n \\
\hline $\mathrm{SiO}_{2}$ & 36.53 & 35.96 & 36.18 & 35.98 & 35.94 & 35.83 & 35.75 & 35.57 & 35.18 & 37.19 & 37.42 & 37.36 & 37.17 & 36.98 & 36.86 \\
\hline $\mathrm{TiO}_{2}$ & 1.59 & 1.48 & 1.71 & 1.72 & 1.45 & 2.26 & 2.13 & .94 & 2.17 & 24 & 1.6 & 2.27 & 6 & 2.09 & 1.9 \\
\hline $\mathrm{Al}_{2} \mathrm{O}_{3}$ & 18.2 & 17.89 & 17.46 & 17.64 & 17.87 & 17.95 & 17.47 & 17.47 & 16.93 & 16.76 & 17.05 & 19.08 & 19.28 & 18.38 & 18.42 \\
\hline $\mathrm{Cr}_{2} \mathrm{O}_{3}$ & 0.00 & 0.00 & 0.00 & 0.00 & 0.00 & 0.00 & 0.00 & 0.00 & 0.00 & 0.00 & 0.00 & 0.00 & 0.00 & 0.00 & 0.00 \\
\hline $\mathrm{Fe}_{2} \mathrm{O}_{3}$ & 0.00 & 0.10 & 0.00 & 0.00 & 0.00 & 0.00 & 0.00 & 0.00 & 0.00 & 0.94 & 0.00 & 0.00 & .00 & 0.00 & 0.00 \\
\hline $\mathrm{FeO}$ & 15.98 & 16.28 & 16.43 & 17.77 & 17.59 & 17.6 & 19.4 & 20.34 & 20.6 & 15.19 & 15.98 & 15.04 & 14.83 & 16.33 & 16.18 \\
\hline $\mathrm{MnO}$ & 01 & 0.00 & 0.01 & 0.12 & 014 & 0 & 0.16 & 0.17 & 0. & 6 & 0.15 & 0.00 & .00 & 0.05 & 0.00 \\
\hline $\mathrm{MgO}$ & 12.23 & 12.4 & 12.29 & 11.24 & 11.7 & 11.16 & 9.14 & 9.1 & 9.23 & 13.53 & 13.15 & 12.26 & 12.9 & 12.04 & 12.3 \\
\hline $\mathrm{CaO}$ & 0.00 & 0.04 & 0.01 & 0.00 & 0.00 & 0.00 & 0.00 & 0.00 & 0.00 & 0.01 & 0.00 & 0.00 & 0.00 & 0.00 & 0.00 \\
\hline $\mathrm{Na}_{2} \mathrm{O}$ & 0.28 & 0.19 & 0.3 & 0.12 & 0.14 & 0.16 & 0.1 & 0.09 & 0.08 & 0.05 & 0.09 & 0.19 & 0.18 & 0.15 & 0.16 \\
\hline $\mathrm{K}_{2} \mathrm{O}$ & 1 & 9 & 8 & 9 & 9.55 & 9.33 & 9.6 & 9.78 & 0 & 9.43 & 9.29 & 9.52 & 9.26 & 9.28 & 2 \\
\hline Sum & 93.55 & 93.42 & 93.24 & 94.12 & 94.39 & 94.39 & 93.76 & 94.46 & 93.44 & 94.51 & 94.75 & 95.75 & 94.89 & 95.3 & 95.14 \\
\hline $\mathrm{Si}$ & 2.77 & 2.747 & 2.767 & 2.755 & 2.742 & 2.731 & 2.773 & 2.756 & 2.753 & 2.802 & 2.809 & 2.758 & 2.762 & 2.761 & 2.756 \\
\hline $\mathrm{Ti}$ & 0.091 & 0.085 & 0.098 & 0 & 0. & 0. & 4 & 13 & 28 & 0 & 09 & 126 & 71 & 117 & .107 \\
\hline $\mathrm{Al}$ & 1.627 & 1.611 & 1.575 & 1.593 & 1.607 & 1.612 & 1.598 & 1.595 & 1.562 & 1.488 & 1.509 & 1.66 & 1.689 & 1.618 & 1.624 \\
\hline $\mathrm{Cr}$ & 0.00 & 0.00 & 0.00 & 0.00 & 0.00 & 0.00 & 0.00 & 0.00 & 0.00 & 0.00 & 0.00 & 0.00 & 0.00 & 0.00 & 0.00 \\
\hline $\mathrm{Fe}^{3+}$ & 0.00 & 0.006 & 0.00 & 0.00 & 0.00 & 0.00 & 0.00 & 0.00 & 0.00 & 0.053 & 0.00 & 0.00 & 0.00 & 0.00 & 0.00 \\
\hline $\mathrm{Fe}^{2+}$ & 1.013 & 1.04 & 1.051 & 1.138 & 1.123 & 1.122 & 1.258 & 1.318 & 1.348 & 0.957 & 1.004 & 0.929 & 0.921 & 1.02 & 1.012 \\
\hline $\mathrm{Mn}$ & 0.001 & 0.00 & 0.00 & 0.008 & 0.009 & 0.006 & 0.01 & 0.011 & 0.011 & 0.01 & 0.01 & 0.00 & 0.00 & 0.003 & 0 \\
\hline $\mathrm{Mg}$ & 1.382 & 1.411 & 1.401 & 1.283 & 1.33 & 1.268 & 1.057 & 1.05 & 1.076 & 1.519 & 1.471 & 1.349 & 1.428 & 1.34 & 1.37 \\
\hline $\mathrm{Ca}$ & 0.00 & 0.003 & 0.001 & 0.00 & 0.00 & 0.00 & 0.00 & 0.00 & 0.00 & 0.001 & 0.00 & 0.00 & 0.00 & 0.00 & 0.00 \\
\hline $\mathrm{Na}$ & 0.042 & 0.028 & 0.045 & 0.018 & 0.021 & 0.023 & 0.016 & 0.013 & 0.012 & 0.007 & 0.012 & 0.028 & 0.026 & 0.022 & 0.023 \\
\hline K & 0.844 & 0.885 & 0.862 & 0.93 & 0.931 & 0.908 & 0.951 & 0.967 & 0.908 & 0.907 & 0.891 & 0.898 & 0.879 & 0.885 & 0.89 \\
\hline Sum & 7.769 & 7.816 & 7.801 & 7.823 & 7.847 & 7.799 & 7.787 & 7.824 & 7.798 & 7.814 & 7.797 & 7.748 & 7.776 & 7.766 & 7.781 \\
\hline $\mathrm{Mg} / \mathrm{Fe}+\mathrm{Mg}$ & 0.577 & 0.576 & 0.571 & 0.530 & 0.542 & 0.531 & 0.457 & 0.443 & 0.444 & 0.613 & 0.594 & 0.592 & 0.608 & 0.568 & 0.575 \\
\hline
\end{tabular}

Formula proportions based on 11 oxygen atoms; $n$ : core and b: rim

MA-3-37 and PO-130, pyrope content is higher than spessartine content. On sample PO-101, the garnet grains composition shows dominance of almandine and spessartine. The profile indicates a discrete zonation, with an increase of Fe and $\mathrm{Ca}\left(\mathrm{alm}_{\mathrm{N} 58.2 \rightarrow \mathrm{B} 59.25}\right.$ and $\left.\mathrm{grs}_{\mathrm{N} 9.84 \rightarrow \mathrm{B} 10.46}\right)$ and decrease of $\mathrm{Mn}$ and $\mathrm{Mg}\left(\mathrm{sps}_{\mathrm{N} 19.08 \rightarrow \mathrm{B} 18.4}\right.$ and $\left.\operatorname{prp}_{\mathrm{N} 12.86 \rightarrow \mathrm{B} 12.4}\right)$ from core to rim (Fig. 5A). Grains in sample PO-153 show increasing Fe from cores to rims $\left(\mathrm{alm}_{\mathrm{N} 60.6 \rightarrow \mathrm{B} 66.0}\right)$ and decreasing $\mathrm{Mn}\left(\mathrm{sps}_{\mathrm{N} 20.6 \rightarrow \mathrm{B} 15.1}\right)$. $\mathrm{Mg}$ content in crystals of this sample has little variation from core to $\operatorname{rim}\left(\operatorname{prp}_{\mathrm{N}_{14.7 \rightarrow B 13.6}}\right)$. Grossular content shows weak compositional banding on the core, suffering a slight decrease $\left(\operatorname{grs}_{\mathrm{N} 4.1 \rightarrow 0.2}\right)$, followed by an increase toward the rims $\left(\mathrm{grs}_{B 3,2 \rightarrow 4.0}\right.$ ) (Fig. 5B).

Sample PO-163 presents the garnet porphyroblasts with dominant composition of almandine, followed by spessartine, pyrope and grossular. It exhibits a chemical

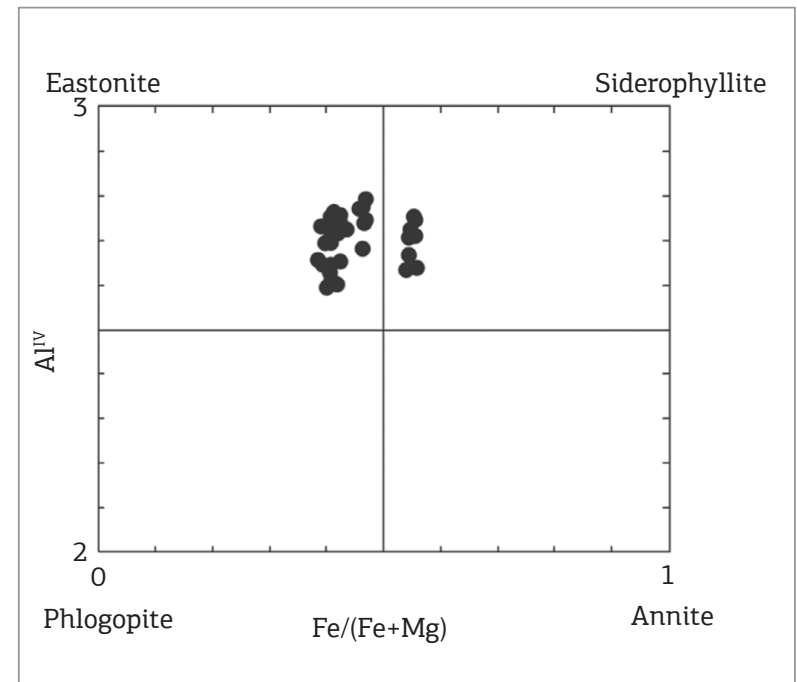

Figure 4. Classification diagram of biotite crystals. 
Table 4. Chemical analysis of staurolite crystals

\begin{tabular}{|c|c|c|c|c|c|c|c|c|c|c|c|c|c|}
\hline Sample & $\begin{array}{l}\hat{n} \\
\stackrel{n}{\dot{1}} \\
\sum\end{array}$ & $\begin{array}{l}\hat{n} \\
\hat{n} \\
\sum \\
\sum\end{array}$ & $\begin{array}{c}\hat{n} \\
\hat{n} \\
\sum \\
\Sigma\end{array}$ & $\begin{array}{c}\hat{n} \\
\hat{n} \\
\dot{\Sigma}\end{array}$ & $\begin{array}{l}\hat{n} \\
\hat{n} \\
\dot{1} \\
\sum\end{array}$ & $\begin{array}{c}\hat{n} \\
\hat{n} \\
\sum \\
\Sigma\end{array}$ & $\begin{array}{c}\hat{n} \\
\hat{n} \\
\sum \\
\Sigma\end{array}$ & $\begin{array}{c}\hat{n} \\
\hat{n} \\
\sum \\
\Sigma\end{array}$ & 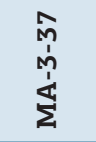 & $\begin{array}{l}\hat{n} \\
\hat{n} \\
\sum \\
\Sigma\end{array}$ & 命 & $\begin{array}{c}\hat{n} \\
\hat{n} \\
\sum \\
\Sigma\end{array}$ & 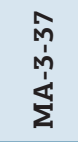 \\
\hline position & $\mathbf{b}^{*}$ & $\mathrm{n}^{*}$ & $\mathrm{n}^{*}$ & $\mathbf{b}^{*}$ & $\mathbf{b}^{* *}$ & $\mathrm{n}^{* *}$ & $\mathrm{n}^{* *}$ & $\mathbf{b}^{* *}$ & $\mathrm{n}^{* *}$ & $\mathbf{b}^{* *}$ & $\mathrm{n}^{* *}$ & $\mathbf{b}^{* *}$ & $\mathrm{n}^{* *}$ \\
\hline $\mathrm{SiO}_{2}$ & 27.73 & 28.00 & 34.08 & 34.02 & 26.88 & 27.71 & 27.60 & 26.84 & 27.45 & 27.66 & 27.90 & 27.21 & 27.80 \\
\hline $\mathrm{TiO}_{2}$ & 1.01 & 0.49 & 0.27 & 0.48 & 0.47 & 0.65 & 0.59 & 0.54 & 0.63 & 0.41 & 0.54 & 0.41 & 0.68 \\
\hline $\mathrm{Al}_{2} \mathrm{O}_{3}$ & 51.47 & 52.13 & 48.32 & 49.13 & 53.68 & 52.40 & 52.87 & 53.74 & 53.52 & 53.64 & 52.84 & 52.90 & 52.60 \\
\hline $\mathrm{Cr}_{2} \mathrm{O}_{3}$ & 0.00 & 0.00 & 0.00 & 0.00 & 0.00 & 0.00 & 0.00 & 0.00 & 0.00 & 0.00 & 0.00 & 0.00 & 0.00 \\
\hline $\mathrm{Fe}_{2} \mathrm{O}_{3}$ & 0.00 & 0.00 & 0.00 & 0.00 & 0.00 & 0.00 & 0.00 & 0.00 & 0.00 & 0.00 & 0.00 & 0.00 & 0.00 \\
\hline $\mathrm{FeO}$ & 14.54 & 14.63 & 14.30 & 13.67 & 14.32 & 14.67 & 14.14 & 14.36 & 13.83 & 13.66 & 13.88 & 14.58 & 14.12 \\
\hline $\mathrm{MnO}$ & 0.11 & 0.06 & 0.09 & 0.08 & 0.08 & 0.13 & 0.15 & 0.15 & 0.13 & 0.13 & 0.17 & 0.14 & 0.17 \\
\hline $\mathrm{MgO}$ & 2.25 & 2.20 & 2.11 & 2.07 & 2.08 & 2.13 & 2.17 & 1.96 & 2.10 & 2.10 & 2.20 & 2.22 & 2.11 \\
\hline $\mathrm{CaO}$ & 0.04 & 0.01 & 0.00 & 0.04 & 0.01 & 0.00 & 0.02 & 0.03 & 0.04 & 0.02 & 0.03 & 0.02 & 0.02 \\
\hline $\mathrm{Na}_{2} \mathrm{O}$ & 0.03 & 0.03 & 0.05 & 0.01 & 0.04 & 0.04 & 0.00 & 0.03 & 0.00 & 0.05 & 0.03 & 0.01 & 0.04 \\
\hline $\mathrm{K}_{2} \mathrm{O}$ & 0.00 & 0.03 & 0.00 & 0.00 & 0.02 & 0.02 & 0.00 & 0.01 & 0.01 & 0.00 & 0.00 & 0.00 & 0.01 \\
\hline Sum & 97.17 & 97.59 & 99.23 & 99.52 & 97.59 & 97.74 & 97.53 & 97.67 & 97.71 & 97.66 & 97.59 & 97.48 & 97.54 \\
\hline $\mathrm{Si}$ & 7.79 & 7.83 & 9.26 & 9.19 & 7.51 & 7.74 & 7.71 & 7.50 & 7.64 & 7.69 & 7.77 & 7.62 & 7.76 \\
\hline $\mathrm{Ti}$ & 0.21 & 0.10 & 0.06 & 0.10 & 0.10 & 0.14 & 0.12 & 0.11 & 0.13 & 0.09 & 0.11 & 0.09 & 0.14 \\
\hline $\mathrm{Al}$ & 17.06 & 17.18 & 15.48 & 15.65 & 17.69 & 17.26 & 17.40 & 17.70 & 17.55 & 17.58 & 17.35 & 17.47 & 17.31 \\
\hline $\mathrm{Cr}$ & 0.00 & 0.00 & 0.00 & 0.00 & 0.00 & 0.00 & 0.00 & 0.00 & 0.00 & 0.00 & 0.00 & 0.00 & 0.00 \\
\hline $\mathrm{Fe}^{3+}$ & 0.00 & 0.00 & 0.00 & 0.00 & 0.00 & 0.00 & 0.00 & 0.00 & 0.00 & 0.00 & 0.00 & 0.00 & 0.00 \\
\hline $\mathrm{Fe}^{2+}$ & 3.42 & 3.42 & 3.25 & 3.09 & 3.35 & 3.43 & 3.30 & 3.36 & 3.22 & 3.18 & 3.23 & 3.42 & 3.30 \\
\hline $\mathrm{Mn}$ & 0.03 & 0.01 & 0.02 & 0.02 & 0.02 & 0.03 & 0.04 & 0.04 & 0.03 & 0.03 & 0.04 & 0.03 & 0.04 \\
\hline $\mathrm{Mg}$ & 0.94 & 0.92 & 0.86 & 0.83 & 0.87 & 0.89 & 0.90 & 0.82 & 0.87 & 0.87 & 0.91 & 0.93 & 0.88 \\
\hline $\mathrm{Ca}$ & 0.01 & 0.00 & 0.00 & 0.01 & 0.00 & 0.00 & 0.01 & 0.01 & 0.01 & 0.01 & 0.01 & 0.01 & 0.01 \\
\hline $\mathrm{Na}$ & 0.02 & 0.02 & 0.03 & 0.01 & 0.02 & 0.02 & 0.00 & 0.02 & 0.00 & 0.03 & 0.02 & 0.01 & 0.02 \\
\hline $\mathrm{K}$ & 0.00 & 0.01 & 0.00 & 0.00 & 0.01 & 0.01 & 0.00 & 0.01 & 0.00 & 0.00 & 0.00 & 0.00 & 0.00 \\
\hline Sum & 29.47 & 29.49 & 28.96 & 28.89 & 29.56 & 29.51 & 29.47 & 29.55 & 29.46 & 29.45 & 29.45 & 29.56 & 29.46 \\
\hline
\end{tabular}

*Staurolite crystals included in garnet porphyroblasts. ${ }^{* *}$ Staurolite crystals on the matrix. Formula proportions based on 46 oxygen atoms; $\mathrm{n}$ : core and b: rim

zonation marked by decreasing on Fe content from core towards the rim $\left(\mathrm{alm}_{\mathrm{N} 69.6-670.6 \rightarrow \mathrm{B} 54.0-63.0}\right)$. Mg content slightly increases on core towards the rim followed by decrease on the rim $\left(\operatorname{prp}_{\mathrm{N} 9.30-10.2 \rightarrow \mathrm{B} 4.9-8.7 .7}\right)$. Mn decreases on the core $\left(\mathrm{sps}_{\mathrm{N} 16.1 \rightarrow 14.2}\right)$ and increases towards the rim $\left(\mathrm{sps}_{\mathrm{B} 23.7}\right)$, followed by decrease along the rim $\left(\mathrm{sps}_{\mathrm{B1} 4.8}\right)$ (Fig. $\left.5 \mathrm{C}\right)$. On sample MA-3-37, Fe shows little compositional variance when comparing core and rims $\left(\mathrm{alm}_{67.0 \rightarrow 66.1}\right)$. Mn content also shows little variance from core towards the rim $\left(\mathrm{sps}_{\mathrm{N} 11.6-12.5 \rightarrow \mathrm{B} 12.2}\right)$, followed by abrupt decrease along the $\operatorname{rim}\left(\operatorname{sps}_{7.0-7.6}\right)$. The $\mathrm{Mg}$ content decreases from the core to the rim $\left(\operatorname{prp}_{\mathrm{N}_{1655-17.2 \rightarrow \mathrm{B} 13.9}}\right)$ (Fig. 5D). In PO-130 sample, composition of garnet crystals has predominance of almandine, followed by pyrope, spessartine and grossular. Profile shows a slight depletion followed by enrichment of $\mathrm{Fe}\left(\operatorname{alm}_{\mathrm{N} 70.0 \rightarrow \mathrm{B} 75.8}\right)$ and $\mathrm{Mg}\left(\operatorname{prp}_{\mathrm{N} 14.3 \rightarrow \mathrm{B} 17.3}\right)$ from core to rim. Ca shows slight enrichment from core to rims followed by depletion along the rims $\left(\mathrm{grs}_{\mathrm{N} 3.4-6.8 \rightarrow \mathrm{B} 4.3}\right)$ and a decrease in $\mathrm{Mn}$ from core to rim $\left(\mathrm{sps}_{\mathrm{N} 11.79 \rightarrow \mathrm{B} 4.3}\right)$ (Fig. $\left.5 \mathrm{E}\right)$.

\section{Muscovite}

Table 6 has the results of chemical analysis of muscovite crystals, which have 5.55 to 5.68 a.p.f.u. of $\mathrm{Si}, \mathrm{Al}^{\mathrm{IV}} / \mathrm{Al}^{\mathrm{VI}}$ ratio between 0.84 and $0.97, \mathrm{X}_{\mathrm{Mg}}$ between 0.31 and 0.55 , and $\mathrm{Si} / \mathrm{Al}$ ratio ranging between 1.07 and 1.16 , being classified as phengite.

\section{Plagioclase}

Plagioclase grains from samples PO-101, PO-153 and $\mathrm{PO}-163$ present homogeneous chemical composition (Tab. 7), with predominant constitution of oligoclase (Fig. 6) with values between $\mathrm{an}_{12.04 \rightarrow 21.7}$, without apparent compositional variance when comparing the grains from the core to the rims. Analyzed crystals on the samples MA-3-37 and PO-130 exhibit compositional variance between oligoclase and andesine $\left(\mathrm{an}_{22.0 \rightarrow 33.9}\right)$, without showing concentric chemical zonation, with domains of different compositions on the crystals (Tab. 7 and Fig. 6). Feldspar grains included in 
Table 5. Chemical analysis and structural formulae of garnet crystal (MA-3-37)

\begin{tabular}{|c|c|c|c|c|c|c|c|c|c|c|c|}
\hline Sample & $\begin{array}{l}\hat{n} \\
\hat{n} \\
\stackrel{1}{\xi} \\
\sum\end{array}$ & $\begin{array}{l}\hat{n} \\
\hat{n} \\
\stackrel{\Sigma}{\Sigma} \\
\sum\end{array}$ & $\begin{array}{l}\hat{m} \\
\hat{n} \\
\stackrel{1}{\vdots} \\
\sum\end{array}$ & $\begin{array}{l}\hat{m} \\
\hat{n} \\
\dot{\Sigma} \\
\sum\end{array}$ & $\begin{array}{l}\hat{n} \\
\hat{n} \\
\stackrel{\Sigma}{\Sigma} \\
\sum\end{array}$ & $\begin{array}{c}\hat{n} \\
\hat{n} \\
\stackrel{\Sigma}{\Sigma}\end{array}$ & $\begin{array}{c}\hat{n} \\
\hat{n} \\
\sum \\
\sum\end{array}$ & $\begin{array}{l}\hat{n} \\
\hat{n} \\
\stackrel{\Sigma}{\Sigma} \\
\sum\end{array}$ & $\begin{array}{c}\hat{n} \\
\hat{n} \\
\stackrel{\Sigma}{\Sigma}\end{array}$ & $\begin{array}{l}\hat{m} \\
\hat{n} \\
\dot{\Sigma} \\
\sum\end{array}$ & $\begin{array}{l}\hat{n} \\
\hat{n} \\
\stackrel{\Sigma}{\Sigma} \\
\sum\end{array}$ \\
\hline position & b & b & b & b & b & $\mathrm{n}$ & b & b & b & b & b \\
\hline $\mathrm{SiO}_{2}$ & 37.33 & 37.13 & 36.95 & 36.61 & 36.94 & 37.18 & 37.06 & 36.90 & 36.91 & 37.26 & 37.04 \\
\hline $\mathrm{TiO}_{2}$ & 0.00 & 0.04 & 0.00 & 0.09 & 0.00 & 0.00 & 0.00 & 0.00 & 0.00 & 0.02 & 0.00 \\
\hline $\mathrm{Al}_{2} \mathrm{O}_{3}$ & 21.91 & 21.89 & 22.13 & 21.86 & 21.87 & 21.89 & 21.79 & 21.51 & 21.64 & 21.61 & 21.66 \\
\hline $\mathrm{Cr}_{2} \mathrm{O}_{3}$ & 0.04 & 0.00 & 0.00 & 0.02 & 0.00 & 0.00 & 0.00 & 0.00 & 0.37 & 0.00 & 0.00 \\
\hline $\mathrm{Fe}_{2} \mathrm{O}_{3}$ & 0.28 & 0.00 & 0.00 & 0.00 & 0.00 & 0.00 & 0.00 & 0.00 & 0.00 & 0.00 & 0.00 \\
\hline $\mathrm{FeO}$ & 29.54 & 27.91 & 29.46 & 29.15 & 28.48 & 28.62 & 28.50 & 28.69 & 29.36 & 29.96 & 29.38 \\
\hline $\mathrm{MnO}$ & 3.35 & 5.17 & 5.35 & 4.95 & 5.39 & 4.90 & 5.40 & 5.00 & 5.35 & 3.49 & 3.09 \\
\hline $\mathrm{MgO}$ & 3.49 & 3.70 & 4.10 & 4.34 & 4.18 & 3.96 & 4.22 & 4.16 & 3.53 & 3.47 & 3.44 \\
\hline $\mathrm{CaO}$ & 4.28 & 2.44 & 1.51 & 1.49 & 1.41 & 1.58 & 1.68 & 1.46 & 2.13 & 3.35 & 4.24 \\
\hline $\mathrm{Na}_{2} \mathrm{O}$ & 0.00 & 0.00 & 0.00 & 0.00 & 0.00 & 0.00 & 0.00 & 0.00 & 0.00 & 0.00 & 0.00 \\
\hline $\mathrm{K}_{2} \mathrm{O}$ & 0.00 & 0.00 & 0.00 & 0.00 & 0.00 & 0.00 & 0.00 & 0.00 & 0.00 & 0.00 & 0.00 \\
\hline Sum & 100.24 & 98.28 & 99.50 & 98.50 & 98.26 & 98.14 & 98.65 & 97.72 & 99.28 & 99.15 & 98.86 \\
\hline $\mathrm{Si}$ & 2.96 & 2.99 & 2.96 & 2.96 & 2.98 & 3.00 & 2.98 & 3.00 & 2.97 & 2.99 & 2.98 \\
\hline $\mathrm{Ti}$ & 0.00 & 0.00 & 0.00 & 0.01 & 0.00 & 0.00 & 0.00 & 0.00 & 0.00 & 0.00 & 0.00 \\
\hline $\mathrm{Al}$ & 2.05 & 2.08 & 2.09 & 2.08 & 2.08 & 2.08 & 2.07 & 2.06 & 2.05 & 2.05 & 2.05 \\
\hline $\mathrm{Cr}$ & 0.00 & 0.00 & 0.00 & 0.00 & 0.00 & 0.00 & 0.00 & 0.00 & 0.02 & 0.00 & 0.00 \\
\hline $\mathrm{Fe}^{3+}$ & 0.02 & 0.00 & 0.00 & 0.00 & 0.00 & 0.00 & 0.00 & 0.00 & 0.00 & 0.00 & 0.00 \\
\hline $\mathrm{Fe}^{2+}$ & 1.96 & 1.88 & 1.97 & 1.97 & 1.92 & 1.93 & 1.92 & 1.95 & 1.98 & 2.01 & 1.98 \\
\hline $\mathrm{Mn}$ & 0.23 & 0.35 & 0.36 & 0.34 & 0.37 & 0.34 & 0.37 & 0.34 & 0.37 & 0.24 & 0.21 \\
\hline $\mathrm{Mg}$ & 0.41 & 0.44 & 0.49 & 0.52 & 0.50 & 0.48 & 0.51 & 0.50 & 0.42 & 0.42 & 0.41 \\
\hline $\mathrm{Ca}$ & 0.37 & 0.21 & 0.13 & 0.13 & 0.12 & 0.14 & 0.15 & 0.13 & 0.18 & 0.29 & 0.37 \\
\hline $\mathrm{Na}$ & 0.00 & 0.00 & 0.00 & 0.00 & 0.00 & 0.00 & 0.00 & 0.00 & 0.00 & 0.00 & 0.00 \\
\hline $\mathrm{K}$ & 0.00 & 0.00 & 0.00 & 0.00 & 0.00 & 0.00 & 0.00 & 0.00 & 0.00 & 0.00 & 0.00 \\
\hline Sum & 8.00 & 7.96 & 8.00 & 8.00 & 7.98 & 7.96 & 7.99 & 7.98 & 7.99 & 7.99 & 8.00 \\
\hline \%Alm & 66.15 & 65.11 & 66.78 & 66.55 & 65.93 & 67.05 & 65.30 & 66.67 & 67.03 & 68.15 & 66.67 \\
\hline \%Prp & 13.92 & 15.37 & 16.56 & 17.65 & 17.22 & 16.56 & 17.23 & 17.21 & 14.35 & 14.06 & 13.90 \\
\hline$\%$ Sps & 7.62 & 12.22 & 12.26 & 11.43 & 12.66 & 11.63 & 12.53 & 11.77 & 12.38 & 8.03 & 7.09 \\
\hline$\%$ Grs & 11.34 & 7.30 & 4.40 & 4.31 & 4.19 & 4.76 & 4.94 & 4.35 & 5.09 & 9.76 & 12.35 \\
\hline$\% A d r$ & 0.82 & 0.00 & 0.00 & 0.00 & 0.00 & 0.00 & 0.00 & 0.00 & 0.00 & 0.00 & 0.00 \\
\hline \%Uvt & 0.14 & 0.00 & 0.00 & 0.05 & 0.00 & 0.00 & 0.00 & 0.00 & 1.16 & 0.00 & 0.00 \\
\hline $\mathrm{Fe} / \mathrm{Fe}+\mathrm{Mg}$ & 82.61 & 80.90 & 80.13 & 79.04 & 79.29 & 80.19 & 79.13 & 79.48 & 82.37 & 82.89 & 82.75 \\
\hline
\end{tabular}

Alm: almandine; Prp: pyrorpe; Sps: spessartine; Grs: grossular; Adr: andradite; Uvt: uvarovite; Formula proportions based on 12 oxygen atoms; n: core and b: rim

staurolite porphyroblasts (sample MA-3-37) show composition similar to feldspar crystals from the matrix, varying between compositional fields of oligoclase and andesine (Tab. 7 and Fig. 6).

\section{METAMORPHISM AND THERMOBAROMETRY}

The calculations of metamorphic P-T conditions were obtained with THERMOCALC software (Powell \& Holland 1994), using composition next to the rims of garnet grains and the most representative ones for other minerals. Obtained results are displayed on Tab. 8.
At Pontalina region, calculated temperature and pressure for gneiss samples ranges from $580 \pm 33^{\circ} \mathrm{C}$ to $628 \pm 17^{\circ} \mathrm{C}$ and $6.7 \pm 0.8 \mathrm{kbar}$ to $8.6 \pm 0.6 \mathrm{kbar}$, respectively, compatible with amphibolite facies in kyanite-staurolite field.

Petrographic analysis of sample MA-3-37 shows staurolite inclusions in the core of garnet crystal and kyanite inclusions on the rims, evidencing progressive metamorphism by appearance of staurolite followed by kyanite (Fig. 7A). On the other hand, presence of subhedral to anhedral staurolite crystals on the matrix, with better external contours than kyanite (Fig. 7B), suggests a new growth phase of staurolite, now posterior to kyanite, evidencing regressive metamorphism, sin-kinematic, in accordance with acquired thermobarometric data, and interpreted 


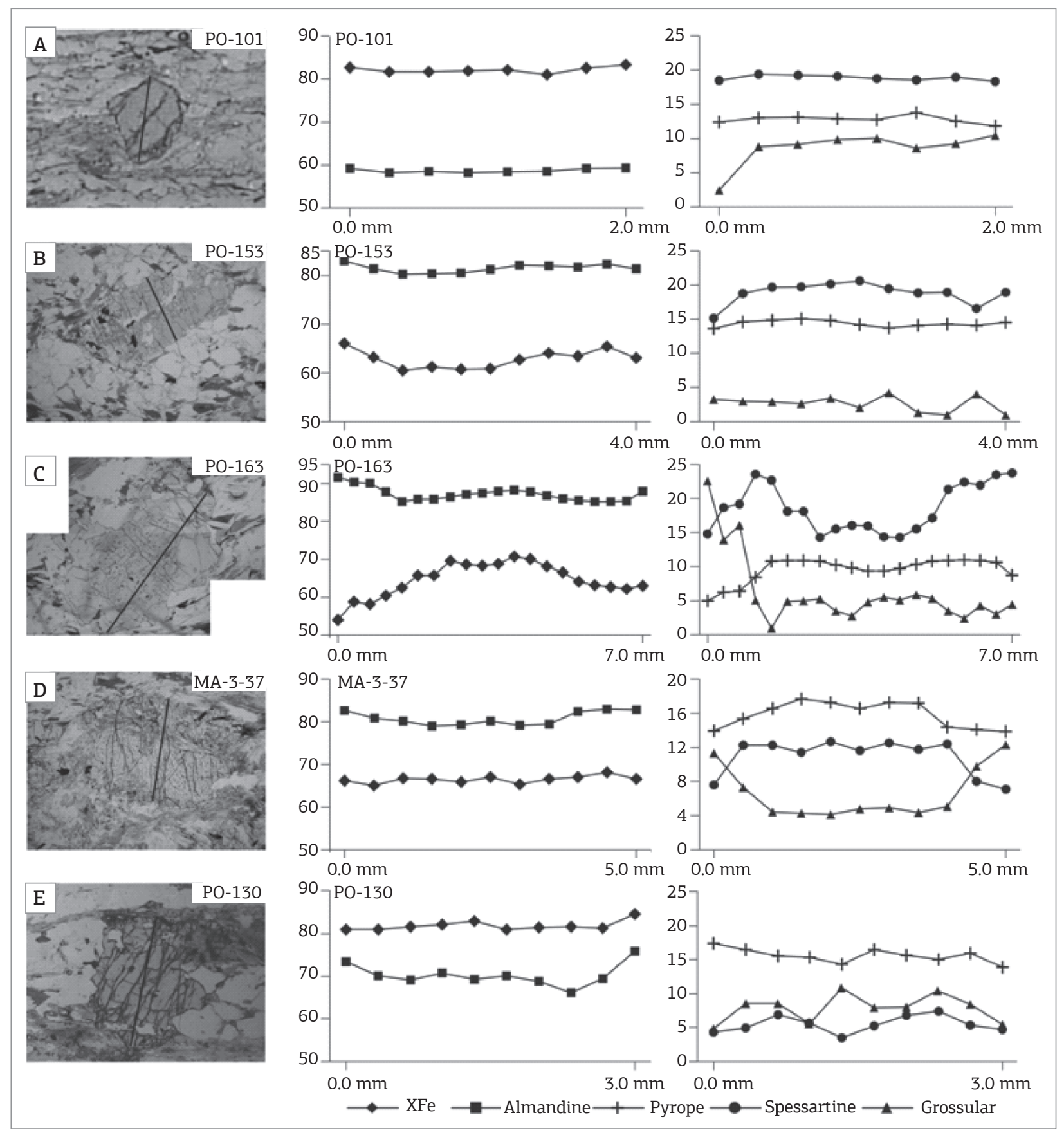

Figure 5. Photomicrography of garnet crystals showing the position of the profiles: PO-101 (A), PO-153 (B), PO163 (C), MA-3-37 (D) and PO-130 (E).

based on petrographic analysis of other samples. This fact is attested by data displayed in Fig. 8, which also suggest a clockwise trajectory for metamorphic evolution of the rocks on this region.

Calculated P-T conditions for these samples are lower than calculated thermobarometric data for amphibole + plagioclase and plagioclase + garnet + amphibole assemblages in metamafic/metabasic rocks, which indicate metamorphic peak at intermediate to high amphibolite facies, reaching temperatures from 700 to $775^{\circ} \mathrm{C}$, in intermediate pressure conditions ( $\sim 10 \mathrm{kbar}$ ) (Navarro et al. 2011). Under these conditions, metamorphic features on metamafic/metabasic rocks are likely to represent regional metamorphic peak, and that mineralogical associations registered on these rocks represent a retrometamorphic equilibrium, also in amphibolite facies, but under milder conditions. This difference can be explained based on strain (schistification) more pronounced on analyzed rocks 
Table 6. Chemical analyses of muscovite crystals

\begin{tabular}{|c|c|c|c|c|c|c|c|c|c|c|c|c|c|c|c|c|}
\hline ample & $\begin{array}{l}\hat{n} \\
\hat{n} \\
\stackrel{1}{\xi} \\
\sum\end{array}$ & $\begin{array}{l}\hat{n} \\
\hat{n} \\
\dot{1} \\
\sum\end{array}$ & $\begin{array}{l}\hat{m} \\
\hat{n} \\
\sum \\
\Sigma\end{array}$ & $\begin{array}{l}\hat{n} \\
\hat{n} \\
\stackrel{\Sigma}{\Sigma}\end{array}$ & $\begin{array}{l}\stackrel{M}{M} \\
\text { ⿳亠丷 } \\
\text { Oे }\end{array}$ & $\begin{array}{l}\text { Mે } \\
\text { †े } \\
\text { Oे }\end{array}$ & $\begin{array}{l}\stackrel{0}{m} \\
\text { ⿳亠丷厂 } \\
\text { Oे }\end{array}$ & $\begin{array}{l}\stackrel{0}{m} \\
\text { †े } \\
\text { Ò }\end{array}$ & 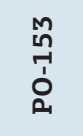 & 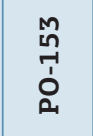 & 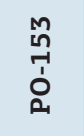 & 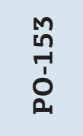 & $\begin{array}{l}\text { no } \\
\text { †1 } \\
\text { o }\end{array}$ & $\begin{array}{l}\text { no } \\
\text { †े } \\
\text { o }\end{array}$ & $\begin{array}{l}\text { ô } \\
\text { †1 } \\
\text { Oे }\end{array}$ & $\begin{array}{l}\text { no } \\
\text { †1 } \\
\text { o }\end{array}$ \\
\hline possit & b & b & $\mathrm{n}$ & $\mathrm{n}$ & $\mathrm{n}$ & b & $\mathrm{n}$ & b & $\mathrm{n}$ & $\mathbf{n}$ & $\pi$ & b & b & $\pi$ & $\mathbf{n}$ & b \\
\hline $\mathrm{SiO}_{2}$ & 5.85 & 45.81 & 45.87 & 45.63 & 46.60 & 46.49 & 6.13 & 46.27 & 45.35 & 45.73 & 45.49 & 45.13 & 45.57 & 45.77 & 44.73 & 44.96 \\
\hline $\mathrm{TiO}_{2}$ & 21 & 0.37 & 0.42 & 0.47 & 0.04 & 0.01 & 0.07 & 0.05 & 0.66 & 0.73 & 0.71 & 0.95 & 0.87 & 0.73 & 0.68 & 0.80 \\
\hline $\mathrm{Al}_{2} \mathrm{O}_{3}$ & 33.98 & 35.30 & 34.18 & 35.22 & 35.14 & 35.75 & 36.54 & 36.45 & 34.09 & 34.19 & 33.55 & 34.41 & 33.91 & 34.47 & 34.30 & 34.55 \\
\hline $\mathrm{Cr}_{2} \mathrm{O}_{3}$ & 0.00 & 0.00 & 0.00 & 0.00 & 0.00 & 0.00 & 0.00 & 0.00 & 0.00 & 0.00 & 0.00 & 0.00 & 0.00 & 0.00 & 0.00 & 0.00 \\
\hline $\mathrm{Fe}_{2} \mathrm{O}_{3}$ & 37 & 0.00 & 0.00 & 0.00 & 0.00 & 0.00 & 0.00 & 0.00 & 0.89 & 0.00 & 0.57 & 0.00 & 0.00 & 0.00 & 0.00 & 0.00 \\
\hline $\mathrm{FeO}$ & .08 & 1.85 & 2.28 & 2.03 & 1.14 & 1.07 & 0.69 & 0.65 & 1.78 & 2.38 & 1.89 & 2.26 & 1.56 & 1.94 & 1.61 & 1.66 \\
\hline $\mathrm{MnO}$ & Do & 0.00 & 0.00 & 0.00 & 0.05 & 0.00 & 0.05 & 0.00 & 0.00 & 0.00 & 0.01 & 0.01 & 0.00 & 0.01 & 0.03 & 0.00 \\
\hline $\mathrm{IgO}$ & 34 & 53 & 0.74 & 61 & 0.80 & 0.6 & 33 & 0.25 & 0.91 & 84 & 0.96 & 0.78 & .03 & 0.94 & 0.93 & 0.84 \\
\hline $\mathrm{CaO}$ & Do & 0.16 & 0.01 & 02 & 0.03 & 0.00 & 0.02 & 0.02 & 0.00 & 0.00 & 0.00 & 0.00 & 0.00 & .01 & 0.00 & 0.02 \\
\hline $\mathrm{Ja}_{2} \mathrm{O}$ & 71 & 2.22 & 1.67 & 1.99 & 1.14 & 1.2 & 1.28 & 1.33 & 1.3 & 13 & 1.2 & 1.39 & 0.57 & 0.6 & 0.64 & 0.60 \\
\hline $\mathrm{K}_{2} \mathrm{O}$ & 8.24 & 7.30 & 8.74 & 8.43 & 9.28 & 9.36 & 8.78 & 8.55 & 8.97 & 9.25 & 8.80 & 9.13 & 10.05 & 10.47 & 10.19 & 9.87 \\
\hline Sum & 3.29 & 93.55 & 93.91 & 94.41 & 94.23 & 94.61 & 93.90 & 93.57 & 93.97 & 94.50 & 93.23 & 94.06 & 93.57 & 94.95 & 93.10 & 93.30 \\
\hline $\mathrm{Si}$ & 3.11 & 3.07 & 3.10 & 3.06 & 3.11 & 3.09 & 3.08 & 3.09 & 3.06 & 3.08 & 3.09 & 3.05 & 3.09 & 3.07 & 3.06 & 3.06 \\
\hline $\mathrm{Al}$ & 2.71 & 2.79 & 2.72 & 2.78 & 2.77 & 2.81 & 2.87 & 2.87 & 2.72 & 2.71 & 2.69 & 2.74 & 2.71 & 2.73 & 2.76 & 2.77 \\
\hline Sum & 4.0 & 4.0 & 4.0 & 4.0 & 4.0 & 4.0 & 4.0 & 4.0 & 4.0 & 4.0 & 4.0 & 4.0 & 4.0 & 4.0 & 4.0 & 4.0 \\
\hline $\mathrm{Al}$ & 82 & 1.86 & 1.82 & 1.84 & 1.88 & 1.90 & 1.95 & 1.96 & 1.78 & 1.79 & 1.78 & 1.79 & 1.80 & 1.80 & 1.82 & 1.83 \\
\hline $\mathrm{Ti}$ & 01 & 0.02 & 0.02 & 0.02 & 0.00 & .00 & 0.00 & 0.00 & 0.03 & 0.04 & 0.04 & 0.05 & 0.04 & 0.04 & 0.04 & 0.04 \\
\hline $\mathrm{Cr}$ & .00 & 0.00 & 0.00 & 0.00 & 0.00 & 0.00 & 0.00 & 0.00 & 0.00 & 0.00 & 0.00 & 0.00 & 0.00 & 0.00 & 0.00 & 0.00 \\
\hline $\mathrm{Fe}^{3+}$ & 02 & 0.00 & 0.00 & 0.00 & 0.00 & 0.00 & 0.00 & 0.00 & 0.05 & 0.00 & 0.03 & 0.00 & 0.00 & 0.00 & 0.00 & 0.00 \\
\hline $\mathrm{Fe}^{2+}$ & 12 & 0.10 & 0.13 & 0.11 & 0.06 & 0.06 & 0.04 & 0.04 & 0.10 & 0.13 & 0.11 & 0.13 & 0.09 & 0.11 & 0.09 & 0.09 \\
\hline $\mathrm{Mn}$ & 0.00 & 0.00 & 0.00 & 0.00 & 0.00 & 0.00 & 0.00 & 0.00 & 0.00 & 0.00 & 0.00 & 0.00 & 0.00 & 0.00 & 0.00 & 0.00 \\
\hline $\mathrm{Mg}$ & 0.08 & 0.05 & 0.07 & 0.06 & 0.08 & 0.07 & 0.03 & 0.03 & 0.09 & 0.09 & 0.10 & 0.08 & 0.10 & 0.09 & 0.10 & 0.09 \\
\hline Sum & 2.00 & 2 & 2.0 & 2.0 & 2.0 & 2.0 & 2.0 & 2.0 & 2.0 & 2.0 & 2.0 & 2.0 & 2.0 & 2.0 & 2.0 & 2.0 \\
\hline $\mathrm{Ca}$ & 0.05 & 0.04 & 0.04 & 0.03 & 0.02 & 0.03 & 0.02 & 0.03 & 0.05 & 0.05 & 0.06 & 0.05 & 0.03 & 0.04 & 0.05 & 0.05 \\
\hline $\mathrm{Na}$ & 0.22 & 0.29 & 0.22 & 0.26 & 0.15 & 0.16 & 0.17 & 0.17 & 0.17 & 0.18 & 0.16 & 0.18 & 0.08 & 0.08 & 0.08 & 0.08 \\
\hline K & 0.71 & 0.63 & 0.75 & 0.72 & 0.79 & 0.80 & 0.75 & 0.73 & 0.77 & 0.80 & 0.76 & 0.79 & 0.87 & 0.90 & 0.89 & 0.86 \\
\hline Sum & 0.98 & 0.96 & 1.01 & 1.01 & 0.96 & 0.99 & 0.94 & 0.93 & 0.99 & 1.03 & 0.98 & 1.02 & 0.98 & 1.02 & 1.02 & 0.99 \\
\hline Sum T & 6.99 & 6.97 & 7.01 & 7.02 & 6.97 & 6.98 & 6.94 & 6.92 & 7.00 & 7.02 & 6.98 & 7.02 & 6.98 & 7.02 & 7.01 & 6.99 \\
\hline
\end{tabular}

Formula proportions based on 11 oxygen atoms; $n$ : core and $b$ : rim

than on the metabasic rocks, due to rheological differences, fact that would have destroyed the rebalancing of greater degrees in gneissic rocks and metasedimentary rocks, and only partially on amphibolitic rocks.

Thus, thermobarometric data acquired on this work reinforce considerations conducted by Navarro et al. (2011), that suggest the existence of a regional metamorphic peak (termed M1), observed on metamafic/ metabasic rocks, from intermediate to high amphibolite facies, on kyanite stability field, and that temperatures around $580-628^{\circ} \mathrm{C}$ and pressures of $6.68-8.6 \mathrm{kbar}$ represent a tectonic-metamorphic rebalancing (M2) of regional expression, on amphibolite facies, staurolite-kyanite field (Fig. 8). Furthermore, in metaultramafic rocks, pseudomorphs and vestiges of olivine are rarely observed, with fabrics suggesting metamorphic reequilibrium in amphibolite facies, and usually the recognized paragenesis and associations are composed of chlorite, serpentine, talc, magnetite and carbonate. Major crystals of chlorite and talc, especially when exhibiting crystallographic orientation, may be generated in retrometamorphic conditions on amphibolite facies, but textural features of the association constituted by serpentine, carbonate, talc, chlorite and magnetite are consistent with greenschist facies, with temperatures below $500^{\circ} \mathrm{C}$, when compared with changes that occurred in the host rocks.

Minerals associated to parageneses at metamorphic peak mark the main foliation. Inclusions observed in garnet porphyroblasts draw shapes that suggest rotation, without showing continuity with the external foliation, which indicates that they grew in earlier or initial stages of the main ductile deformational phase (Dn). Retrometamorphism is 
Table 7. Representative chemical analysis of plagioclase

\begin{tabular}{|c|c|c|c|c|c|c|c|c|c|c|c|c|c|c|c|}
\hline e & $\begin{array}{l}\hat{n} \\
\hat{n} \\
\stackrel{1}{\sum} \\
\sum\end{array}$ & $\stackrel{n}{\stackrel{n}{\infty}}$ & 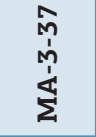 & 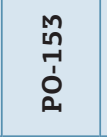 & 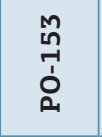 & 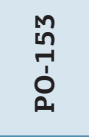 & 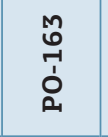 & $\begin{array}{l}\text { ô } \\
\text { ज̇ } \\
\text { ó }\end{array}$ & $\begin{array}{l}\text { ô } \\
\text { ì } \\
\text { o }\end{array}$ & $\begin{array}{l}\text {-1 } \\
\text {-1 } \\
\text { ó } \\
\text {. }\end{array}$ & $\begin{array}{l}-1 \\
0 \\
7 \\
0 \\
0\end{array}$ & $\begin{array}{l}\text { - } \\
\text { ने } \\
\text { ó }\end{array}$ & 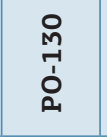 & 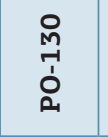 & $\begin{array}{l}\stackrel{M}{M} \\
\stackrel{1}{\circ} \\
\text { م. }\end{array}$ \\
\hline & $n$ & $n$ & b & b & b & $n$ & b & $n$ & $\mathrm{n}$ & b & $\mathrm{n}$ & b & $\mathrm{n}$ & $\mathrm{n}$ & b \\
\hline $\mathrm{SiO}_{2}$ & 0.31 & 9.83 & 0.50 & 3.80 & 3.32 & 2.32 & 3.15 & 3.68 & 2.56 & 3.12 & 3.22 & 3.17 & 9.92 & 9.85 & 0.41 \\
\hline $\mathrm{TiO}_{2}$ & 0.02 & 0.00 & 0.00 & 0.00 & 0.00 & 0.00 & 0.00 & 0.00 & 0.00 & 0.06 & 0.00 & 0.01 & 00 & 0.00 & 0.04 \\
\hline $\mathrm{Al}_{2} \mathrm{O}_{3}$ & .11 & .26 & 25.19 & 23.51 & 23.52 & 23.59 & 23.28 & 23.58 & 23.80 & 23.25 & 23.14 & 23.01 & 5.52 & 25.20 & 25.39 \\
\hline $\mathrm{Cr}_{2} \mathrm{O}_{3}$ & 0 & .00 & 0.00 & 00 & 00 & 0.00 & 0.00 & 0.00 & .00 & 0.00 & 0.00 & 0.00 & 0.00 & 0.00 & 0.00 \\
\hline $\mathrm{Fe}_{2} \mathrm{O}_{3}$ & 19 & 00 & 0 & 16 & 17 & 17 & 0.06 & 0.03 & 08 & 0.06 & 0.05 & 0.00 & 0.04 & .17 & 0.00 \\
\hline $\mathrm{MnO}$ & 2 & 22 & 2 & 0.02 & 3 & 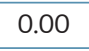 & 0.00 & 0.00 & 01 & 0.00 & 0.05 & 0.05 & . & 0.00 & 0.01 \\
\hline $\mathrm{MgO}$ & 0 & & 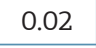 & 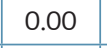 & 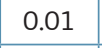 & 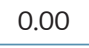 & 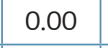 & 0.00 & .00 & 0.00 & 0.01 & 0.00 & 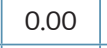 & 0 & 0.01 \\
\hline $\mathrm{CaO}$ & 23 & 6.45 & 19 & $\bar{C}$ & 38 & 38 & 4 & 08 & 4.36 & 7 & 4.54 & 4. & 6 & 6.96 & 6.89 \\
\hline $\mathrm{Na}_{2} \mathrm{O}$ & 78 & 81 & 7.94 & 9.20 & 9.19 & 9.45 & 9.43 & 8.77 & 13 & 9.35 & 9.36 & 9.10 & 7.77 & 8.10 & 7.94 \\
\hline $\mathrm{K}_{2} \mathrm{O}$ & 10 & 05 & 0.06 & 0.11 & 0.06 & 0.07 & 0.07 & 0.09 & 10 & 0.06 & 0.05 & 0.06 & 0.05 & 0.04 & 0.09 \\
\hline Sum & 99.77 & 99.43 & 100.22 & 100.95 & 100.65 & 99.99 & 100.03 & 100.23 & 100.05 & 100.17 & 100.42 & 99.84 & 100.57 & 100.32 & 100.76 \\
\hline $\mathrm{Si}$ & 2.69 & 2.68 & 2.69 & 2.79 & 2.78 & 2.76 & 2.79 & 2.80 & 2.77 & 2.79 & 2.79 & 2.80 & 2.66 & 2.66 & 2.67 \\
\hline $\mathrm{Ti}$ & 0.00 & 00 & 0 & .00 & 00 & 0.00 & 0.00 & 0.00 & .00 & 0.00 & 0.00 & 0.00 & 0.00 & .00 & 0.00 \\
\hline $\mathrm{Al}$ & 2 & & & 1 & & 23 & 1.21 & 22 & 21 & & 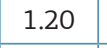 & 1.20 & נכינד & ${ }^{2}$ & 1.32 \\
\hline $\mathrm{Cr}$ & 00 & & & & & 0.00 & & 00 & ( & & 0.00 & 0.00 & 0 & 0 & 0.00 \\
\hline $\mathrm{Fe}^{3+}$ & & & & & & 001 & & & & & P०० & & Pח & & 0 \\
\hline $\mathrm{Mn}$ & 00 & 00 & & & & 0.00 & & 0.00 & & & 0.00 & 0.00 & 0.00 & 0.00 & 0.00 \\
\hline $\mathrm{Mg}$ & & & & & & 0.00 & & & & & 0.00 & 0.00 & 0.00 & 0.00 & 0.00 \\
\hline $\mathrm{Ca}$ & 0.30 & 31 & 1 & 19 & 1 & 0.21 & 0.19 & 0.19 & 21 & 0.20 & 0.22 & 0.21 & 0.35 & .33 & 0.33 \\
\hline $\mathrm{Na}$ & 0.67 & 68 & 068 & 0.78 & 078 & 0.81 & 0.81 & 0.75 & 0.78 & 0.80 & 0.80 & 0.78 & 0.67 & .70 & 0.68 \\
\hline K & 1 & 0.00 & 0.00 & 1 & 0.00 & 0.00 & 0.00 & 0.01 & 0.01 & 0.00 & 0.00 & 0.00 & 0.00 & .00 & 0.01 \\
\hline Sum & 4.99 & 5.00 & 5.00 & 4.99 & 5.00 & 5.03 & 5.01 & 4.97 & 5.01 & 5.01 & 5.01 & 5.00 & 5.01 & 5.02 & 5.01 \\
\hline Albite & 3.92 & 68.45 & 68.64 & 79.61 & 78.93 & 79.30 & 80.48 & 79.13 & 78.61 & 79.60 & 78.61 & 78.41 & 65.75 & 67.60 & 67.26 \\
\hline Anorthite & 30.46 & 31.24 & 31.06 & 19.78 & 20.77 & 20.31 & 19.12 & 20.34 & 20.78 & 20.10 & 21.10 & 21.29 & 33.96 & 32.11 & 32.25 \\
\hline Orthoclase & 0.62 & 0.30 & 0.30 & 0.61 & 0.30 & 0.39 & 0.40 & 0.53 & 0.60 & 0.30 & 0.29 & 0.30 & 0.30 & 0.29 & 0.49 \\
\hline
\end{tabular}

Formula proportions based on 8 oxygen atoms. n: core and b: rim.

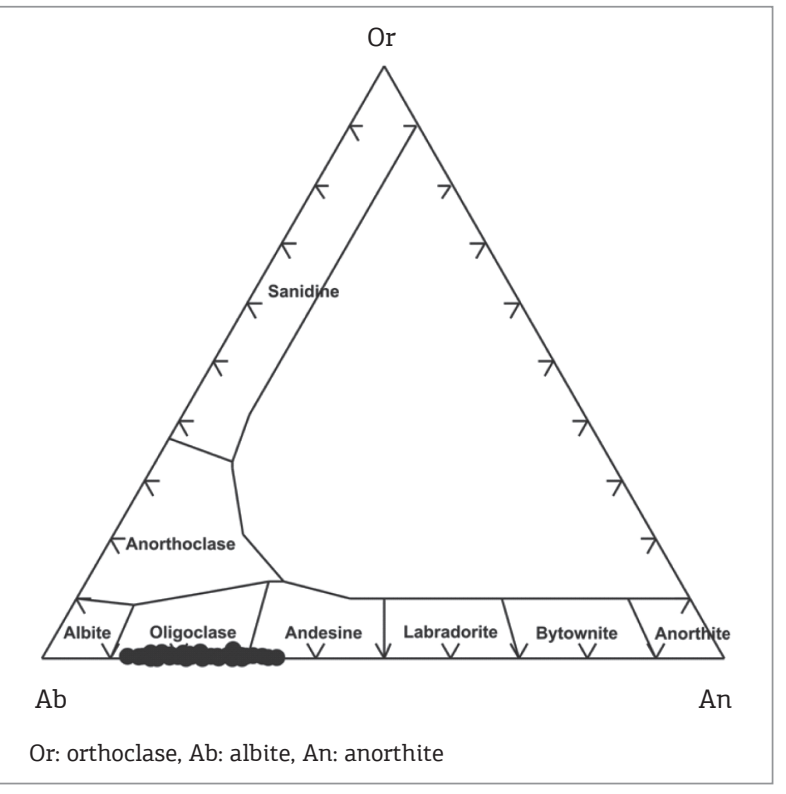

Figure 6. Feldspar classification diagram.
Table 8. Results of P-T conditions of metamorphism, obtained with THERMOCALC

\begin{tabular}{c|c|c|c|c}
\hline Sample & $\mathbf{T}\left({ }^{\circ} \mathbf{C}\right)$ & $\mathbf{2 \sigma}$ & $\mathbf{P}$ (kbar) & $\mathbf{2 \sigma}$ \\
\hline MA-3-37 & 606 & 15 & 7.78 & 0.91 \\
\hline PO-101 & 580 & 33 & 7.90 & 1.10 \\
\hline PO-130 & 628 & 17 & 8.60 & 0.60 \\
\hline PO-153 & 592 & 30 & 6.68 & 0.75 \\
\hline PO-163 & 596 & 19 & 6.91 & 0.73 \\
\hline
\end{tabular}

marked by growth of large muscovite crystals, arranged in aleatory distribution on/above the main foliation, and also by plagioclase saussuritization, kyanite muscovitization and chlorite formation on biotite and garnet, sometimes substituting a significant part of grains. These associations reveal variations in metamorphic conditions from amphibolite facies to an intermediate to upper greenschist facies. Retrometamorphism results of mineral equilibrium sin- to tardi-Dn, since minerals formed in this phase are strained and partially oriented. 


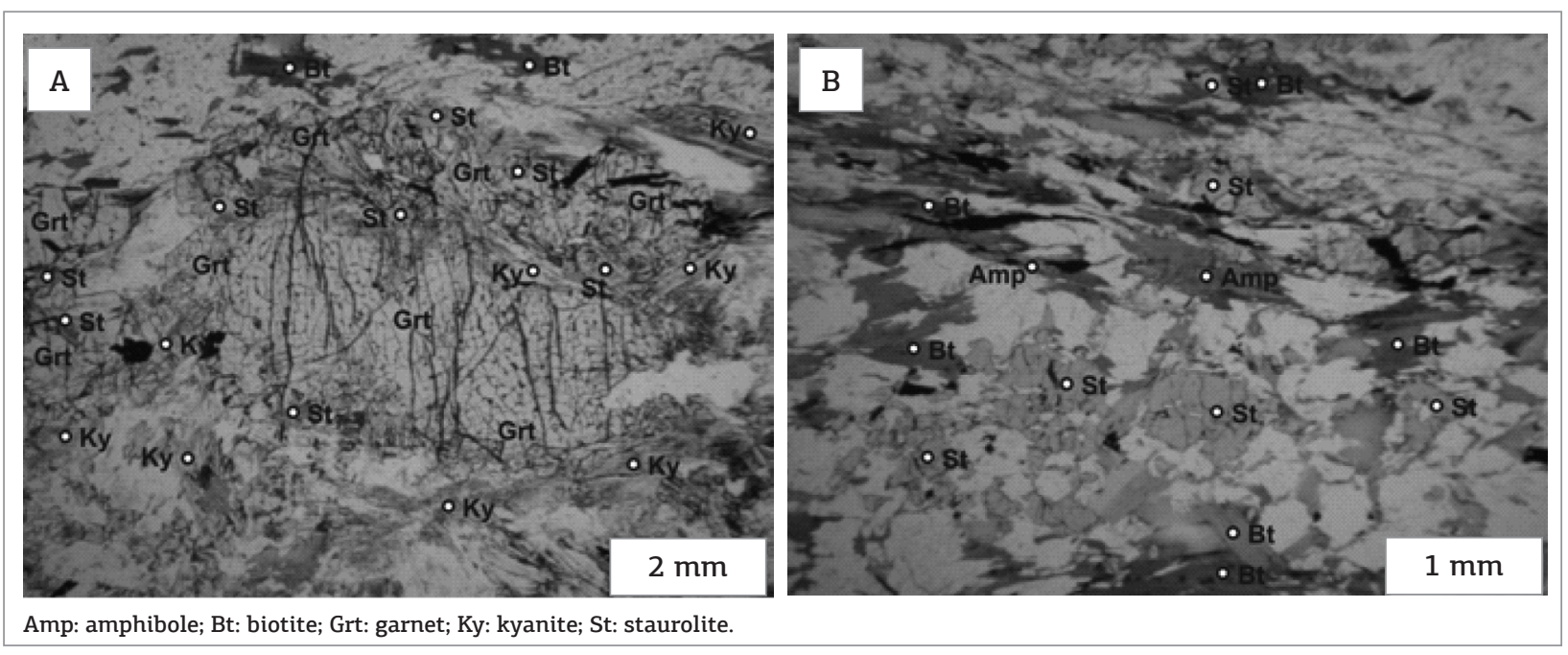

Figure 7. Photomicrography of garnet (Grt) porphyroblast with staurolite (St) inclusions and kyanite (Ky) (sample MA-3-37) (A), and of anhedral staurolite crystals on the matrix (sample MA-3-37) (B).

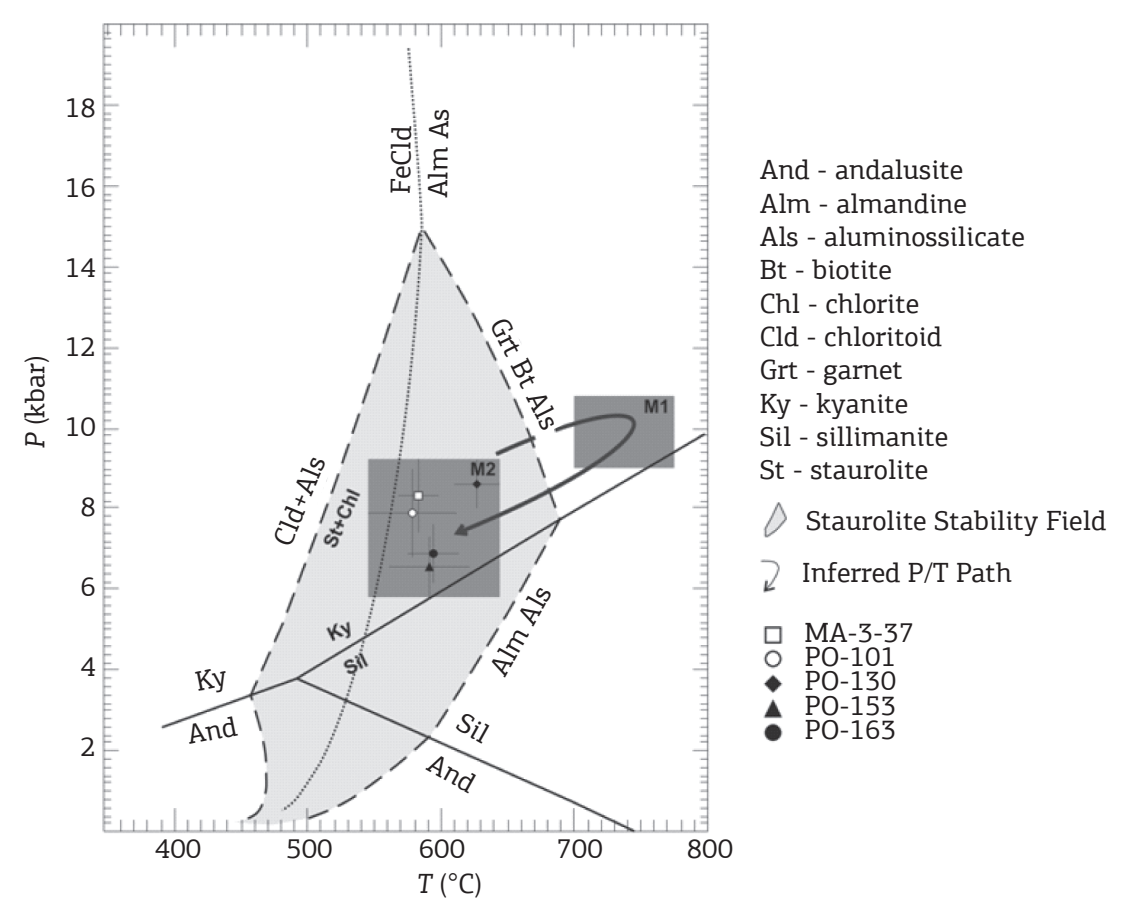

Figure 8. Diagram P x T showing P-T path of metamorphism of Goiás Magmatic Arc and metamorphic gradients of field.

\section{CONCLUSIONS}

The oldest mineral assemblages found in gneissic and metasedimentary rocks from Goiás Magmatic Arc, Pontalina region, which are associated to a metamorphic peak, typically represent amphibolite facies, in kyanite-staurolite field. Thermobarometric calculations obtained for these associations resulted in temperatures of $580-628^{\circ} \mathrm{C}$ and pressures between 6.68 - 8.6 kbar. Petrographic analyses show that reaching of apparent metamorphic peak occurred during formation of the main foliation, meaning it is correlated to deformational phase Dn (regional). These calculated metamorphic conditions, when compared with the ones obtained for the intercalated metamafic/metabasic rocks, show that this is not a regional metamorphic peak, being interpreted as a metamorphic rebalancing (M2) on amphibolite facies, kyanite-staurolite field. Metamorphic peak (M1) would only be preserved in metamafic/metabasic rocks, when compared with the acid terms, assumption sustained by field and petrographic 
observations. In these rocks, thermobarometric calculations result in temperatures between $700-775^{\circ} \mathrm{C}$ and pressures about $10 \mathrm{kbar}$. Conjugate analyses of data obtained from quartz-feldspar gneissic rocks, metasedimentary and metabasic rocks show that P-T path is clockwise, such as observed in other regions of Brasília Belt.

\section{AKNOWLEDGMENTS}

This investigation was performed under a scholarship from FAPESP (Process no 2008/50723-0). The authors thank FAPESP (Process no 2005/59203-1) and CNPq (Process no 301216/2008-8), which contributed with financial support.

\section{REFERENCES}

Barbosa O., Braun O.P.G., Dyer R.C., Cunha C.R. 1970. Projeto Goiânia: relatório preliminar. Goiânia, DNPM-PROSPEC, v.1.

Brod J.A., Leonardos O.H., Meneses P.R., Almeida R., Araújo R.N.J., Blanco S.B., Cardoso F.B.F., Carvalho Jr. O.A., Junqueira F.F., Riehl Jr. W., Souza J.C.F., Tallarico F.H.T., Thomsem F.P.R., Albuquerque M.A.C., Berbet M.L.C., Cerqueira M.R.S., Chagas M.A., Dias R.R., Lima C.V., Nakamura E.T., Porto S.G., Romão P.A., Santos P. C.V. 1991. Tectonoestratigrafia da Faixa Brasília na região do Alto Paranaíba. In: SBG, Simpósio de Geologia do Centro-Oeste, 3., Anais... p. 155-168.

Campos Neto M.C. \& Caby R. 1999. Neoproterozoic High-Pressure Metamorphism and Tectonic Constraint from the Nappe System South of the São Francisco Craton, Southeeast Brazil. Precambriam Research, 97:3-26.

Campos Neto M.C. \& Caby R. 2000. Terrane accretion and upward extrusion of high-pressure granulites in the Neoproterozoic nappes of Southeast Brazil: petrology and structural constraints. Tectonics, 19(4):669-687

Dardenne M.A. 2000. The Brazilia Fold Belt. In: Cordani U.G., Thomas Filho A., Campos D. A. (coords.). Tectonic Evolution of South America. Rio de Janeiro, SBG, p. 231-236.

Del Lama, E.A., Oliveira M.A.F., Zanardo A. 1994. Geotermobarometria em rochas do Complexo Campos Gerais ao norte da Zona de Cisalhamento Varginha. Revista Brasileira de Geociências, 24(4):1-7.

Drake Jr., A.A. 1980. The Serra de Caldas window, Goiás. Tectonic Studies in the Brazilian Shield. Geological Survey of America Professional Paper. Washington, 1119-A, 13 p.

Fischel D.P., Pimentel M.M., Fuck R.A. 1998. Idade do metamorfismo de alto grau no Complexo Anápolis-Itauçu, Goiás, determinada pelo método Sm-Nd. Revista Brasileira de Geociências, 28(4):543-544

Fuck R.A., Jardim de Sá E.F., Pimentel M.M., Dardenne M.A., Pedrosa Soares A.C. 1993. As faixas de Dobramentos Marginais do Cráton do São Francisco: síntese dos conhecimentos. In: Dominguez J.M.L. \& Misi A. (coords.). O Cráton do São Francisco, Salvador, SBG, p. 161-185.

Leake B.E., Woolley A.R., Arps C.E.S., Birch W.D., Gilbert M.C., Grice J.D., Hawthorne F.C., Kato A., Kisch H.J., Krivovichev V.G., Linthout K., Laird Jo, Mandarino J.A., Maresch W.V., Nickel E.H., Rock N.M.S., Schumacher J.C., Smith D.C., Stephenson N.C.N., Ungaretti L., Whittaker E.J.W., Youzhi G. 1997. Nomeclature of amphiboles: Report of the Subcommittee on Amphiboles of the International Mineralogical Association, Commission on New Minerals and Mineral Names. American Mineralogist, 82:1019-1037.

Luvizotto G.L. 2003. Caracterização metamórfica das rochas do Grupo Araxá na região de São Sebastião do Paraíso, Sudoeste de Minas Gerais. Dissertação de Mestrado, Instituto de Geociências e Ciências Exatas, Universidade Estadual Paulista "Júlio de Mesquita Filho", Rio Claro, 193p.

Marini O.J., Fuck R.A., Danni J.C.M., Dardene M.A., Lougguércio R.O., Ramalho R. 1984a. As faixas de dobramentos Brasília, Uruaçu e
Paraguai-Araguaia e o Maciço Mediano de Goiás. In: Schobbenhaus C., Campos D.A., Derze G.R., Asmus H.E. (coords.). Geologia do Brasil. Brasília, DNPM., p. 251-303.

Marini O.J., Fuck R.A., Dardene M.A., Danni J.C.M. 1984b. Províncias Tocantins: setores Central e Sudeste. In: Almeida F.F.M., Hasuy Y. (coords.). O Pré-cambriano do Brasil. São Paulo, Edgar Blücher, p. 205-264.

Moraes R., Brown M., Fuck R.A., Camargo M.A., Lima T.M. 2002. Characterization and P-T evolution of melt-bearing ultrahightemperature granulites: an example from the Anápolis-Itauçu Complex of the Brasília Fold Belt, Brazil. Journal of Petrology, 43(9):1673-1705

Navarro G.R.B. \& Zanardo A. 2006. Dados geoquímicos e isotópicos de rochas metamáficas da região de Pontalina - GO. Revista de Geologia, 19(2):207-214.

Navarro G.R.B. \& Zanardo A. 2007a. Geoquímica de Gnaisses do Arco Magmático de Goiás na região Sul do Estado de Goiás. Geologia USP: Série Científica, 7(1):19-28.

Navarro G.R.B. \& Zanardo A. 2007b. Características geoquímicas de gnaisses da Unidade Metassedimentar, porção sul do Arco Magmático de Goiás, na região de Pontalina. In: Congresso Brasileiro de Geoquímica, 11., 2007. Atibaia, São Paulo. Resumos... (CD-ROM).

Navarro G.R.B., Zanardo A., Simões L.S.A. 2007. Rochas metamáficas associadas ao arco magmático de Goiás na região de Pontalina GO. Revista Brasileira de Geociências, 37(4 Suppl):18-27.

Navarro G.R.B., Moraes R., Zanardo A., Simões L.S.A., Conceição F.T. 2009. Trajetória P-T e condições do metamorfismo usadas como ferramenta para compartimentação tectônica da Faixa Brasília em Goiás. Revista Brasileira de Geociências, 39(3):544-559.

Navarro G.R.B., Zanardo A., Conceição F.T. 2011. Evolução metamórfica e termobarometria das rochas metabásicas da região de Pontalina - Morrinhos, Goiás. Revista Brasileira de Geociências, no prelo.

Nilson A.A. 1984. O atual estágio de conhecimento dos Complexos máfico-ultramáficos pré-cambrianos do Brasil - uma avaliação preliminar. In: SBG, Congresso Brasileiro de Geologia, 33., 1984. Rio de Janeiro. Anais... v. 9, p. 4166-4203.

Oliveira M.A.F., Carvalho S.G., Morales N., Rodrigues M.F.B., Zanardo A. 1983. Geologia das quadrículas de Cássia e São Sebastião do Paraíso - MG. In: Simpósio de Geologia de Minas Gerais, 2., 1983. Belo Horizonte. Anais... Boletim no 3, p. 11-14.

Pimentel M.M., Fuck R.A., Jost H., Ferreira Filho C.F., Araújo S.M. 2000. The basement of the Brasília Fold Belt and the Goiás magmatic arc. In: Cordani U.G., Thomas Filho A., Campos D.A. (coords.) Tectonic Evolution of South America. In: International Geological Congress, 31., 2000. Rio de Janeiro. p. 195-229. 
Pimentel M.M. \& Fuck R. A. 1991. Origin of orthogneiss and metavolcanic rock units in western Goiás: Neoproterozoic crustal accretion. Geochimica Brasiliensis, 5:133-152.

Pimentel M.M. \& Fuck R.A. 1992. Neoproterozoic crustal accretion in central Brazil. Geology, 20:375-379.

Pimentel M.M., Jost H., Fuck R.A. 2004. O embasamento da Faixa Brasília e o arco Magmático de Goiás. In: Mantesso Neto V., Bartorelli A., Carneiro C.D.R., Brito Neves B.B. (orgs.). Geologia do Continente Sul-Americano: evolução da obra de Fernando Flávio Marques de Almeida. São Paulo, Beca, p. 355-368.

Piuzana D., Pimentel M.M., Fuck R.A., Armstrong R. 2003. Neoproterozoic granulite facies metamorphism and coeval granitic magmatism in the Brasília Belt, central Brazil. Precambrian Research, 125:139-273.

Powell R. \& Holland T. 1984. Optimal geothermometry and geobarometry. American Mineralogist, 79:120-144.

Simões L.S.A. 1995. Evolução tectonometamórfica da Nappe de Passos, sudoeste de Minas Gerais. Tese de Doutorado, Instituto de Geociências, Universidade de São Paulo, São Paulo, 149p.

Simões L.S.A., Valeriano C.M., Morales N., Zanardo A., Moraes R., Gomi C.Y. 1998. Zonação metamórfica inversa do Grupo AraxáCanastra na região de São Sebastião do Paraíso-Alpinópolis, MG. In SBG, Congresso Brasileiro de Geologia, 35., 1998. Belém. Anais... v. 3, p. $1203-1215$
Strieder A.J. \& Nilson A.A. 1992. Melange ofiolítica nos metassedimentos Araxá de Abadiânia (GO) e implicações tectônicas regionais. Revista Brasileira de Geociências, 22(2):204-215.

Valeriano C.M., Dardenne M.A., Fonseca M.A., Simões L.S.A., Seer H.J. 2004. A evolução tectônica da Faixa Brasília. In: Mantesso Neto V., Bartorelli A., Carneiro C.D.R., Brito Neves B.B. (orgs.). Geologia do Continente Sul-Americano: evolução da obra de Fernando Flávio Marques de Almeida. São Paulo, Beca, p. 575-592.

Winge M. 1995. Evolução dos terrenos granulíticos da Província Tocantins, Brasil Central. Tese de Doutorado, Instituto de Geociências, Universidade de Brasília, Brasília, 550p.

Zanardo A., Morales N., Carvalho S.G., Simões L.S.A., Oliveira M.A.F. 1990. Evolução metamórfica da porção sul do Cráton do Paramirim. In: SBG, Congresso Brasileiro de Geologia, 36., 1990. Natal. Anais... v. 4, p. 1945-1955.

Zanardo A., Morales N., Del Lama E.A. 1992. Evolução metamórfica do Grupo Araxá-Canastra na região de Passos (MG). In: SBG, Congresso Brasileiro de Geologia, 37., 1992. São Paulo. Anais... v. 1, p. 408-409.

Zanardo A., Oliveira M.A.F., Del Lama E.A., Carvalho S.G. 1996. Geologia do Grupo Araxá de Passos-São Sebastião do Paraíso, sul de Minas Gerais. Geociências, 15(1):253-278.

Arquivo digital disponível on-line no site www.sbgeo.org.br 